\title{
Statistical Curvature Change Analysis of Random- Shape Polyethylene Microplastics and their Toxicity from a Physical Perspective
}

\section{Daheui Choi}

Yonsei University

Jangsun Hwang

Chung-Ang University

Junah Bang

Sungkyunkwan University

Seora Han

Yonsei University

Taeho Kim

Yonsei University

Yoogyeong Oh

Yonsei University

Youngdeok Hwang

City College of New York

Jonghoon Choi

Chung-Ang University

Jinkee Hong ( $\square$ jinkee.hong@yonsei.ac.kr)

Yonsei University https://orcid.org/0000-0003-3243-8536

\section{Research}

Keywords: Polyethylene, Primary microplastics, Secondary microplastics, Morphology, Statistics, Toxicology

Posted Date: May 13th, 2020

DOI: https://doi.org/10.21203/rs.3.rs-28527/v1

License: (c) (1) This work is licensed under a Creative Commons Attribution 4.0 International License.

Read Full License 
3 Daheui Choi ${ }^{a, \S}$, Jangsun Hwang ${ }^{b, \S}$, Junah Bang ${ }^{c}$, Seora Han ${ }^{a}$, Taeho Kim ${ }^{a}$, Yoogyeong Oh $^{a}$,

$4 \quad$ Youngdeok Hwang ${ }^{d}$, Jonghoon Choi ${ }^{b, *}$, and Jinkee Hong ${ }^{a, *}$

6 a Department of Chemical and Biomolecular Engineering, Yonsei University, Seoul 03722,

$7 \quad$ Republic of Korea

$8 \quad{ }^{b}$ School of Integrative Engineering, Chung-Ang University, Seoul 06974, Republic of Korea

$9 \quad{ }^{c}$ Department of Statistics, Sungkyunkwan University, Seoul 03132, Republic of Korea

$10 \mathrm{~d}$ Paul H. Chook Department of Information Systems and Statistics, Baruch College CUNY,

11 New York, NY, United States of America

$12{ }^{\S}$ Both authors contributed equally to this work

*Corresponding authors: Tel.: +82-2-2123-5748; Fax: +82-2-312-6401; E-mail:

14 jinkee.hong@yonsei.ac.kr (Jinkee Hong)

15 Tel.: +82-2-820-5258; Fax: +82-2-814-2651; E-mail: nanomed@cau.ac.kr (Jooghoon Choi) 


\section{Abstract}

\section{Background}

20 Microplastics, plastics that have gradually and randomly decomposed into small fragments after exposure to physical and biological external stress, are emerging as a significant environmental threat. They are normally categorized into the following three types: particles, fibers, and random-shape fragments broken down from bulk plastics. Here, we have demonstrated the in vitro toxicity of microplastics of two different shapes. To minimize the chemical effect, polyethylene (PE), which has a basic chemical polymer structure, was used. PE microplastics with two different shapes were prepared, high-density PE (HDPE) microbeads and randomly ground low-density PE (LDPE) from bulk pellets.

\section{Results}

29 To quantify the randomness of the microplastic shape, the edge patterns of the generated PE microplastics were converted into numerical values and analyzed using a statistical method. A 10-fold difference in curvature value was observed between PE particles and ground PE microfragments. We found that the higher concentration and rough structure were associated with the toxicity of plastics toward immune- or non-immune cells, pro-inflammatory cytokine release, and hemolysis, even though PE is buoyant onto medium. The smooth PE particles did not exhibit severe cytotoxicity at any of the tested concentrations, but induced immune and hemolysis responses at high concentrations.

\section{Conclusion}

When comparing the toxicity of two different shapes of PE microplastics, we confirmed by statistical analysis that random-shape plastics with sharp edges and higher curvature 
40 differences may adversely affect human cells.

41 Keywords: Polyethylene; Primary microplastics; Secondary microplastics; Morphology;

42 Statistics; Toxicology

43 


\section{Background}

The global use of plastics has dramatically increased every year since their development in the mid-1900s; in 2015 alone, 381 million tons of plastic was produced [1]. Over the past several decades, the amount of plastic waste has increased significantly (in the range of millions of tons), and there has been a continuous accumulation of various kinds of plastic waste in oceans, rivers, and landfills [2]. When plastic waste enters an ocean, it gradually breaks down into smaller fragments [1]. Plastics that are less than $5 \mathrm{~mm}$ in size are called microplastics [3]. They can be categorized into the following two types: manufactured smallsize plastics such as microbeads used as exfoliants in cosmetics, or fibers from clothing (primary microplastics); and the breakdown products of bulk plastics (secondary microplastics) [1]. Secondary microplastics can be degraded by exposure to ultraviolet light, wave action, wind, and abrasion [4].

Polyethylene (PE), polypropylene (PP), and polystyrene (PS) are abundant in spherical microplastics, and are mostly found in beach sediments or in the deep sea $[5,6,7]$. Because of their size in the micron-range-different from that of bulk plastic products-these microplastics may exert different effects on the marine biota [8]. Marine organisms, such as mussels or fish, can ingest microplastics, which might then be transferred to humans via the consumption of seafood or sea salts [8,9]. Typically, plastic microdebris readily adsorb various harmful components, including bacteria, viruses, and toxic chemicals (persistent organic pollutants), which might cause unknown diseases upon accumulation in the human body [8]. In addition, microplastics that are broken down into different shapes could have different effects on cells (i.e., toxicity) depending on their sharpness or unevenness. With respect to the physical cytotoxicity of materials, previous investigations on shape- and concentration-dependent toxicity have mainly studied inorganic nanoparticles, such as gold, 
silica oxide, and titanium oxide nanoparticles $[10,11,12,13]$. These studies demonstrated that the cytotoxicity of inorganic nanoparticles can be affected by size, shape, concentration, and surface charge [12]. Additionally, the sharpness of nanomaterials may be another factor affecting cytotoxicity; for example, membranes can be physically punctured by aggregates of single-walled carbon nanotubes [13]. From a material point of view, only the effects of concentration and size (in case of spherical particles) on physical toxicity have been investigated until now $[14,15,16,17]$, however, the effects of different structures, especially the random shape of microplastics, have not been investigated. The shapes of microplastics in real environments are diverse and include spheres, fibers, or random fragments [18]. More information is needed regarding the following: cell damage caused by randomly broken microplastics and the chemical properties of microplastics.

In this study, we demonstrated the cytotoxicity of PE microplastics based on their physical properties (concentration and particle shape) and compared statistical microplastic shape (curvature) data and in vitro toxicological results. To quantify the randomness of the shape of microplastics, curvature differences were defined based on the local curvature, which is defined by the slope differences of the tangent lines (a differential function) in each section of the edge (change rate of the curvature). The statistical analysis of curvature shows the shape differences among microplastics. We also examined the toxicity of PE microplastics using various kinds of cells; these included immune (peripheral blood mononuclear cells [PBMCs], HMC-1 human mast cells, and red blood cells [RBCs], and RBL-2H3 rat basophilic leukemia cells)) as well as non-immune cells (human dermal fibroblasts [HDFs] and HeLa cervical cancer cells). As PE consists of only carbon and hydrogen, with no functional side groups, thereby minimizing chemical toxicity to cells, it was selected as the best candidate microplastic to demonstrate physical cytotoxicity. PE is one of the most commonly used 
plastics, and is mainly used as a packaging material in the form of plastic bags, plastic films, and containers (bulk plastics), and as scrubs in cosmetics (microplastics). PE plastics can be categorized into various types depending on the density and polymeric structure, e.g., highdensity PE (HDPE), low-density PE (LDPE), and linear LDPE (LLDPE).

We used the following two types of PE microplastics: manufactured HDPE microparticles and milled LDPE microfragments, which are regarded as primary and secondary sources of microplastics, respectively (Fig. 1). The random shapes of the PE particles were systematically analyzed to statistically quantify the ruggedness of the fragments using an uncertainty metric, and to correlate these values with cytotoxicity. The objectives of the study were to determine the following: (1) cytotoxicity of the accumulated microplastics (concentrations) and (2) physical damage induced by randomly broken microplastics (particle shapes) via statistical analysis of the unevenness of PE microparticles. To achieve this, we used the following types of cells and assays (Fig. 1. Bottom). (i) Surface roughness of the microplastics was quantified by statistical analysis. The quantified roughness of the microplastics was compared with the results of physical toxicity experiments. (ii) Immune cells (PBMCs and mast cells) are among the most sensitive to external stress and materials, and initiate an immune response in response to infection. Cytokine release by immune cells was used to assess the toxicity of microplastics. (iii) Cancer cells (HeLa and RBL-2H3) and fibroblasts, which are non-immune cells, were used to determine the effect of microplastics on the cell survival rate. (iv) RBCs are present in the blood, but are not immune cells. They can release hemoglobin when exposed to external physical and chemical stressors, which can be easily detected by assessing cell membrane damage. To the best of our knowledge, this is the first study describing the cytotoxic effect exerted by virtue of the physical aspects of two different types of microplastics. 


\section{Methods}

\subsection{Materials and particle characterization}

Clear PE microspheres (density $0.96 \mathrm{~g} / \mathrm{cc}$ ) were purchased from Cospheric LLC (Santa Barbara, CA, USA). We used three different sizes of PE microbeads for analysis, 1-10 $\mu \mathrm{m}$, 45-53 $\mu \mathrm{m}(50 \mu \mathrm{m})$, and 90-106 $\mu \mathrm{m}(100 \mu \mathrm{m})$. The following media were used for culturing specific cells: high-glucose Dulbecco's modified Eagle's medium (DMEM; HDFs and HeLa cells); Iscove's modified Dulbecco's medium (IMDM; HMC-1 cells); RPMI-1640 supplemented with L-glutamine (PBMCs). All supplement media were obtained from Gibco (Waltham, MA, USA). Fetal bovine serum (FBS) was purchased from Welgene (Gyeongsansi, Gyeongsangbuk-do, Korea). $\mathrm{Mg}^{+}$and $\mathrm{Ca}^{2+}$-free $10 \times$ phosphate buffered saline $(\mathrm{PBS})$ and penicillin-streptomycin (PS) were procured from Gibco. RBC lysis buffer was purchased from Merck (Darmstadt, Germany). Lipopolysaccharide (LPS) from Escherichia coli and 10\% Triton X-100 were procured from Sigma-Aldrich (St. Louis, MO, USA). The sizes of HDPE and LDPE particles were measured by field-emission scanning electron microscopy (SEM) on IT-500HR (JEOL, Tokyo, Japan).

\subsection{Preparation of LDPE microfragments}

LDPE pellets were crushed using a hammer. The crushed pellets were then chopped into small pieces $(0.2-0.3 \mathrm{~cm})$ using scissors. Pieces of LDPE and zirconia balls were immersed in liquid nitrogen for 10 min to allow for an increase in the hardness of LDPE. After 10 min, the frozen LDPE pieces and zirconia balls were put into a mini ball mill (Fritsch, IdarOberstein, Germany) and milled for $5 \mathrm{~min}$ at $45 \mathrm{rpm}$. The milling process was repeated 10 times. To sort LDPE fragments into different sizes, the ground LDPE fragments were sequentially sifted using $25-\mu \mathrm{m}, 75-\mu \mathrm{m}$, and $200-\mu \mathrm{m}$ mesh size sieves. The filtered particles 
were treated with methanol to disperse individual particles, followed by drying overnight.

\subsection{PBMC culture}

Uncharacterized human PBMCs were purchased from C. T. L. (Shaker Heights, OH, USA). PBMCs were obtained from randomly selected Hispanic or Asian donors (30-50 years of age). The PBMC culture medium contained 90\% RPMI-1640 supplemented with Lglutamine, $10 \%$ heat-inactivated FBS (inactivated at $56^{\circ} \mathrm{C}$ for $30 \mathrm{~min}$ ), and $1 \%$ PS. PBMCs were cultured in an incubator containing an atmosphere of $5 \% \mathrm{CO}_{2}$ for one day at a density of $1 \times 10^{7}$ cells per T25 flask (SPL Life Science, Pocheon-si, Gyeonggi-do, Korea).

\subsection{Live-dead staining and viability analysis}

PBMCs were incubated for one day, and the cells were pelleted at $330 \times g$ for $7 \mathrm{~min}$. The PBMCs were treated with RBC lysis buffer buffer (Roche, Mannheim, Germany) for 5 min before seeding. After RBC elimination, PBMCs were seeded $\left(1 \times 10^{5}\right.$ cells $/ 0.5 \mathrm{~mL} /$ well $)$ in 24-well plates (SPL) and cultured for one day. Doubly concentrated suspension of microparticles $(2 \mathrm{mg} / 0.5 \mathrm{~mL}, 200 \mu \mathrm{g} / 0.5 \mathrm{~mL}$ and $20 \mu \mathrm{g} / 0.5 \mathrm{~mL}$ ) was added to the PBMC without media elimination. As a positive control, cells were treated with $1 \%$ Triton X-100. After one day, the 24-well plate was centrifuged at $330 \times g$ for $7 \mathrm{~min}$ to pellet the cells, and the supernatant was carefully aspirated. The Live/Dead cell viability assay kit for mammalian cells (Thermo Fisher Scientific, Waltham, MA, USA) was used to analyze cell viability. Cells were diluted in $1 \times$ PBS according to the manufacturer's protocol and were treated for $30 \mathrm{~min}$ in the dark. Live (green)/dead (red) images were observed by fluorescence microscopy (Leica, Wetzlar, Germany). PBMC viability was deduced by calculating the ratio of live:dead cells. Cell viability $(\%)$ was calculated and normalized as follows $(\mathrm{n}=3)$ : 
161 Cell viability $(\%)=\frac{\text { Live cells }}{\text { Total cells }} \times 100$

\subsection{Cytokine release}

PBMCs were seeded $\left(5 \times 10^{5}\right.$ cells/ $0.5 \mathrm{~mL} /$ well $)$ in a 24 -well plate. The cells were treated with microplastics as mentioned above and incubated at $37^{\circ} \mathrm{C}$ in an incubator containing a $5 \%$ $\mathrm{CO}_{2}$ atmosphere for 4 days. To measure the cytokine release-including that of interleukin (IL)-2, IL-6, and tumor necrosis factor-alpha (TNF- $\alpha$ )—from the PBMCs, the culture supernatant was collected by centrifugation, followed by detection using enzyme-linked immunosorbent assay (ELISA; BioLegend, San Diego, CA, USA). Maximum immune response upon treatment with $5 \mu \mathrm{g} / \mathrm{mL}$ LPS was used as a positive control.

\subsection{HMC-1 culture}

The HMC-1 cell line was obtained from Merck. The complete HMC-1 growth medium consisted of 90\% IMDM, 10\% heat-inactivated FBS, and 1\% PS. HMC-1 cells were cultured at a density of $2 \times 10^{5}$ cells $/ \mathrm{mL}$ in $100 \mathrm{~mm}$ culture dish at an atmosphere containing $5 \% \mathrm{CO}_{2}$, and were harvested at a density of $2 \times 10^{6}$ cells $/ \mathrm{mL}$.

\subsection{Histamine release}

HMC-1 cells were seeded at a density of $1 \times 10^{5}$ cells/wells of a 96-well plate. After one day of seeding, cells were treated with microbeads and incubated for 2 days. The supernatant was collected, and the amount of histamine released from cells was analyzed by histamine ELISA (MyBioSource, San Diego, CA, USA) (n=3).

\subsection{Hemolysis test}

Sheep blood (MBcell) cells were washed with PBS and centrifuged at 2,000 rpm for 5 min to 
remove hemolyzed RBCs. This process was repeated until a clear supernatant was obtained after centrifugation. The blood was further diluted using PBS, and cells were seeded in a 96well plate. The cells were treated with equal volumes of microparticles suspended in cell growth medium. The blood-microplastic mixture was incubated with mild agitation at 500 rpm for $1 \mathrm{~h}$. Hemolysis was analyzed in the supernatant at an absorbance of $450 \mathrm{~nm}$ after centrifuging $(2,000 \mathrm{rpm}, 7 \mathrm{~min})$ the culture; cells treated with $1 \%$ Triton $\mathrm{X}-100$ were used as a positive control.

\subsection{HeLa, HDF, and RBL-2H3 culture and CCK-8 assay}

HeLa, HDF, and RBL-2H3 cells were cultured in a $100 \mathrm{~mm}$ dish (SPL) in a $5 \% \mathrm{CO}_{2}$ incubator for 2-3 days until they reached $95 \%$ confluence. The cells were then detached from the culture dish by trypsinization and seeded in wells of a 96-well plate at a density of $3 \times 10^{3}$ cells/well. Twenty-four hours later, the growth medium was aspirated, and fresh medium containing the microplastic suspension was added. After $24 \mathrm{~h}$ of treatment, the microbeads were removed and 10\% CCK-8 reagent (Dojindo, Kumamoto, Japan) was added to the cells. The absorbance was measured at $450 \mathrm{~nm}$ using a SpectraMax $340 \mathrm{PC}$ plate reader (Molecular Devices, San Jose, CA, USA). Cells treated with $10 \%$ dimethylsulfoxide (DMSO) or $1 \%$ Triton X-100 were used as positive controls.

\subsection{ROS assay}

The ROS assay kit was purchased from Sigma-Aldrich. HDF cells were seeded in a 96-well plate at a density of $6 \times 10^{5}$ cells/mL. After $24 \mathrm{~h}, 2^{\prime}, 7^{\prime}$-dichlorodihydrofluorescein diacetate (DCFH-DA; $100 \mu \mathrm{L}$ ) was added to each well and incubated for $2 \mathrm{~h}$. DCFH-DA-treated HDF cells were washed twice with PBS and treated with different concentrations of ground LDPE 
particles for $6 \mathrm{~h}$ to induce ROS release. ROS-producing cells emitted a fluorescent signal that was detected using a fluorescence reader at excitation and emission wavelengths of $480 \mathrm{~nm}$ and $530 \mathrm{~nm}$, respectively. Cells treated with $1 \mathrm{mM}$ hydrogen peroxide were used as a positive control for ROS generation.

\subsection{Statistical analysis}

The numerical data shown in the graphs represent the mean value with standard deviation (error bar). Statistical analysis was performed by one-way analysis of variance (ANOVA) followed by post-hoc statistical analysis (Tukey's test). $p$-value $>0.05$ was considered nonsignificant (ns). *,**, and $* * *$ indicate $p<0.05, p<0.01$, and $p<0.001$, respectively. All the experiments were conducted at least three times.

\subsection{Statistical analysis of microplastic images}

\subsubsection{Local curvature}

In a two-dimensional space, the local curvature $(c)$ is defined as

$$
c=\frac{\left|x^{\prime} y^{\prime \prime}-x^{\prime \prime} y\right|}{\sqrt{\left|\left(x^{\prime}\right)^{2}+\left(y^{\prime}\right)^{2}\right|^{3}}}
$$

where $x$ and $x$ are the first and second derivatives in the horizontal direction, respectively, and $y^{\prime}$ and $y$ are similarly defined. For shape analysis, the coordinates were rotated so that the downward direction in Euclidean space is toward the center of the debris when calculating the local curvature at every pixel. Local curvature values were obtained at each pixel around the boundary. A negative curvature value at a boundary pixel indicated that the boundary is bent outward (e.g., a complete circle has a constant negative value reciprocal of 

the radius) at the pixel, whereas a positive value meant that it is bent inward (i.e., dent). Hence, frequent changes in curvature over the boundary were interpreted as being more rugged.

\subsubsection{Hypotheses test}

It is difficult to make a typical assumption in statistical hypotheses, such as normality or independence sampling in our setting, because of the spatial dependence inherent in microscopic images. Thus, we used a nonparametric test that requires minimal assumptions and conditions. Our test relies on the resampling procedure. For simplicity, we assume that the same number of sample images are obtained for both the plastic types, although it can be flexibly chosen with minimal modification. The procedure is summarized below.

1. For each sample $(i=1, \ldots, I)$ and type of the plastic $j=1$ (HDPE), 2(LDPE), randomly pick a partition of length $K$. Denote this partition $y_{i j}^{(b)}=\left(y_{i j 1}^{(b)}, y_{i j 2}^{(b)}, \ldots, y_{i j K}^{(b)}\right)$.

2. Within this partition, obtain the length $K-1$-vector containing the change at each point, denoted by $\bar{y}_{i 1}^{(b)}$ and $\bar{y}_{i 2}^{(b)}$, respectively.

3. Obtain the average curvature change for $j$, denoted by $\bar{y}_{i}^{(b)}=\frac{1}{I} \frac{1}{K-1} \sum_{i=1}^{I} \sum_{k=1}^{K-1}\left|\tilde{y}_{i j k}^{(b)}\right|$, and define $\bar{w}^{(b)}=\left(\bar{y}_{1}^{(b)}, \bar{y}_{2}^{(b)}\right)$.

After repeating 1-3 B times, $\bar{w}^{(1)}, \ldots, \bar{w}^{(B)}$ were obtained. These statistics can be used for other inferences, such as hypothesis testing or constructing a confidence interval. In this study, we focused on comparing the average curvature for different plastic types. Specifically, we conducted a hypothesis test with the following hypotheses:

$\mathrm{H}_{0}$ : HDPE and LDPE have the same mean curvature change. 
$\mathrm{H}_{1}$ : HDPE and LDPE have a different mean curvature change.

246 Apparently, this test is not available in the "over-the-shelf" statistical software. We created an 247 R package termed 'microplastics' for analysis. This package can be installed and is publicly 248 available at a webpage along with a detailed description 249 (http://ydhwang.github.io/supplement/microplastic_hdpe_ldpe_suppl.html).

\section{Results and discussion}

\subsection{Characterization of PE microplastics}

In the in vitro assays performed using HDPE and LDPE microparticles (Fig. 1), we observed the behavior of various cell types including immune-related cells, RBCs, cancer cells, and fibroblasts. Fig. 2 and 3 show the SEM images and size distribution of the HDPE and LDPE microparticles are mainly used as exfoliants in cosmetics (scrubs) and toothpastes. SEM and size distribution graphs revealed that these particles were highly spherical in shape and showed a narrow size distribution. HDPE particles $>50 \mu \mathrm{m}$ showed uneven surface profiles (Fig. S1).

To obtain LDPE microparticles, LDPE pellets were broken by ball milling to mimic the secondary source of microplastics, which are usually derived from degradation of bulk plastics. The bulk PE waste is likely to physically yield random shape of microparticles. As shown in Fig. 3, LDPE particles ranging from $25-75 \mu \mathrm{m}$ and $75-200 \mu \mathrm{m}$ in size were 
prepared. Both particle sizes displayed a random morphology. However, the larger particles had a sheet-like shape with slightly smooth faces and a random edge plane. The area distribution of both particles was very broad and was calculated to be 6 times higher for the particles in the size range $75-200 \mu \mathrm{m}\left(10560 \mu \mathrm{m}^{2}\right)$ than for those in the size range $25-75 \mu \mathrm{m}$ $\left(1571 \mu \mathrm{m}^{2}\right)$. LDPE is defined structurally as an irregular polymer with random branches and voids [20], and HDPE has a linear structure with fewer branches. These structural differences are attributed to the general physical properties of plastics, resulting in the low crystallinity, low melting temperature, and low hardness of LDPE [21, 22]. Therefore, the ground edge of LDPE appeared slightly blunt because of the softness of LDPE plastics. However, the production of ball-milled HDPE from the pellets proved more difficult. PE has a low brittle temperature, typically $-76{ }^{\circ} \mathrm{C}$ [23]. A low ductile-brittle transition temperature is required for grinding in liquid nitrogen. HDPE has high crystallinity (approximately 70\%) because of its linear polymer structure and shows brittleness and toughness. The higher crystallinity of HDPE with larger spherulites facilitates the formation of a microvoid around polymers and a precreak tip. Microvoids shield against cracking, particularly under dynamic strain [23]. Microvoid formation depends on several factors, such as the size of spherulites, loading rate, strength or deformability of the amorphous zone, and polymer entanglement loss [23]. The difficulties in the production of HDPE microparticles by ball milling with nitrogen liquid treatment can be attributed to a larger shielding effect. Therefore, we prepared ground LDPE microplastics to compare the toxic effects associated with the physical attributes. Though chemical differences exist between HDPE and LDPE, we assumed that the chemical effect of both particles is negligible because the basic chemical structure of HDPE and LDPE is derived from polyethylene. 


\subsection{Statistical shape and curvature differences analysis of PE microplastics}

SEM indicated that LDPE fragments were more rugged than HDPE particles. However, it was difficult to quantify and compare the physical aspects of both microplastics for cellular analysis. By statistically analyzing microplastic images, we assessed the ruggedness of both microplastics. From the original SEM image, we sampled some of the HDPE and LDPE particles (Fig. 4A). The image size was adjusted to be compared equally, and the image was converted into matrices in order to estimate the curvature along the boundaries (Fig. 4A). We have converted the scalable images into pixels for comparison, and every value is dimensionless. The local curvature, which is the rate change of the curvature, in both particles is presented as a series of local curvature estimates. Local curvatures are estimated for each plastic type (Fig. 4B), where the "Boundary pixel of the microplastic" in the x-axis indicates the converted boundary of the microplastics from Fig. 4A. Rougher LDPE microplastics showed large distribution of curvature around 0 (0 value means absolute spherical particle). LDPE displayed more abrupt change points, indicating more rugged boundaries. Further, LDPE fragments were approximately 10 times more rugged than synthetic HDPE particles (Fig. 4C). We then conducted a hypothesis test using $\bar{w}^{(1)}, \ldots, \bar{w}^{(B)}$, with $K=100$ and $B=5000$. The resulting $p$-value $=0 \approx 0.0001$ supported the conclusion that these two types of plastics have significantly different shapes in terms of the change of local curvature. Therefore, we may conclude that the higher curvature of LDPE fragments can be a factor for inducing an enhanced immune response and cytotoxicity.

\subsection{Cellular survival rate analysis under conditions of PE microplastic treatment}

Before measuring the effect of microplastics on cells, we optimized the microplastic concentration after considering the annual intake of microparticles by humans. Microparticle 
in the range of $100 \mu \mathrm{m}$ in size are abundant in the marine environment. Thus, the weight of a single HDPE particle can be calculated by analyzing the density $(\sim 0.98 \mathrm{~g} / \mathrm{cc})$ and volume; a value of $0.5 \mu \mathrm{g}$ per particle was estimated. According to the WWF (World Wide Fund for Nature), humans ingest an average of approximately 2,000 microplastics per week from water, sea salts, seafood, and alcohols [24], which corresponds to $1000 \mu \mathrm{g}$ of PE microplastics. The ingested microplastics enter the gut, and smaller plastics accumulate in tissues, like the liver or bladder, and are absorbed across the gut [25]. Hence, considering accumulated and ingested microplastics (worth 1 week) in humans, we chose a PE particle concentration $<1000 \mu \mathrm{g} / \mathrm{mL}$ in the experiments. Even though the human body is composed of organs which are many times bigger in size than the cells we used for experiments, we aimed to use a high concentration $(1 \mathrm{mg} / \mathrm{mL})$ to model the damage to cells in the specific organs where microplastics continuously accumulate. Furthermore, both HDPE and LDPE particles are hydrophobic and are highly unstable in deionized water and PBS (data not shown). According to the manufacturer, the PE particles require surfactants, such as Tween-80, to enable dispersal in deionized water. To increase particle dispersion, FBS-supplemented DMEM (cell culture media) - that was slightly hydrophobic because of its amino acid, vitamin, and protein content — was used. Additionally, PE is lighter than water, and therefore floats on the medium. PE particles in culture media are initially well-distributed and then slowly begin to float over a period of time; however, a portion of the plastic is still suspended in the medium. Therefore, we believe that the first initial treatment with PE could result in direct contact with cells and induce physical toxicity. Chemical toxicity could have an impact on cells at all treatment times tested.

Fig. 5, S2, and S3 indicate the viability of PBMCs treated with microplastics measured by live-dead staining. The live/dead staining ratio (viability) revealed that HDPE and a low 
concentration of LDPE particles showed no- or low cytotoxicity. However, at high concentrations LDPE particles affected cell survival, and treatment with 25-75 $\mu \mathrm{m}$ and $75-$ $200 \mu \mathrm{m}$ particles resulted in survival rates of $65 \%$ and $82 \%$, respectively. Furthermore, the viability assays for normal adherent cells showed that HDPE particles were non-toxic to cells at high concentrations. However, slight cell-death was occurred for ground LDPE particle treated group, with a viability of approximately $78.8 \%(25-75 \mu \mathrm{m})$ and $61.7 \%(75-200 \mu \mathrm{m})$ for HDF cells and approximately $90 \%$ for RBL-2H3 at high concentration (1 mg/mL) (Fig. 6).

The typical methods to assess cell damage caused by extrinsic toxic materials include examining the following: cellular membrane rupture by strong positive charge [26], interference with DNA synthesis, or organelle activities after uptake [27], which result in cell death due to necrosis or apoptosis. However, PE particles that are not charged cannot induce cell membrane rupture, and their size (in $\mu \mathrm{m}$ ) hampers their cellular uptake. Therefore, they are less likely to damage nuclei or organelles in the cytosol. Smaller PE particles $(<30 \mu \mathrm{m})$ reportedly inhibit osteoblast proliferation and induce the expression of osteocalcin [28], which in turn promotes the secretion of IL-6 or granulocyte-macrophage colony-stimulating factor (GM-CSF) [29]. Cell mortality due to PE particles was not evident in this study, which is in agreement with our result that smooth PE microparticles (HDPE microplastics) were not cytotoxic in any of the tested cell types at a wide range of concentrations. The main reason for cell toxicity of ground LDPE might be the sharpness of some of the ground edges (Fig. 3) that were apparent in SEM images, and were indicated as being important in the statistical analysis (Fig. 4). It is also possible that cell damage was caused by small broken particles originating from the weakened portions of LDPE microparticles.

\subsection{Immune response to PE microplastics}


We further analyzed the immunological response of PBMCs to HDPE and LDPE particles, which was confirmed by the release of IL- 6 and TNF- $\alpha$. IL- 6 and TNF- $\alpha$ are the main proinflammatory cytokines that exert pleiotropic effects on inflammation, immune response, and hematopoiesis [30]. Both of these cytokines are secreted from infected lesions at an initial stage of inflammation and mediate the synthesis of acute-phase proteins, such as C-reactive protein, serum amyloid A, and fibrinogen in hepatocytes, [31] and activation of endothelial cells to increase vascular permeability in vivo [32, 33]. Furthermore, IL-6 stimulates the differentiation of CD4+ and CD8+ T-cells into mature T-cells, and facilitates the generation of an adaptive immune response during infections. High levels of TNF- $\alpha$ are found in acute and chronic inflammatory diseases and activate and recruit the immune cells, particularly regulating the differentiation or proliferation of macrophages, depending on the presence or absence of certain cytokines, such as macrophage colony-stimulating factor-1 [34].

Lipopolysaccharide (LPS) is an endotoxin consisting of lipid and polysaccharide moieties. LPS is a constituent of the outer membrane of gram-negative bacteria. It promotes an inflammatory response via inducing the secretion of IL- 6 and TNF- $\alpha$. In this study, we used cells treated with $5 \mu \mathrm{g} / \mathrm{mL}$ LPS as positive control. As shown in Fig. 6, LPS-treated cells showed significantly increased levels of released cytokines, which indicated the induction of an immune response in PBMCs. Cells treated with HDPE microparticles displayed cytokine release levels that were similar to the control, except at a high concentration of microplastics (Fig. 7A). In particular, compared to the control, IL-6 and TNF- $\alpha$ release was increased upon microparticle treatment by up to 2.6 times $(1-10 \mu \mathrm{m}, 1 \mathrm{mg} / \mathrm{mL})$ and 1.4 times $(100 \mu \mathrm{m}, 1$ $\mathrm{mg} / \mathrm{mL}$ ). We did not observe a size-dependent increase in the immune response, suggesting that all the HDPE microplastics used in this study were similar (for 1-10 $\mu \mathrm{m}$ ) or much larger 
$(>50 \mu \mathrm{m})$ in size than PBMCs. Furthermore, we observed morphological changes in PBMCs five days after HDPE treatment (Fig. 8). Compared to the original morphology of PBMCs, as indicated in Fig. S2 and S3, microparticle-treated cells had a spindle-like or dendritic cell-like morphology, and were considered to be IL-6 or TNF- $\alpha$ secreting differentiated monocytes [35]. A previous report described the induction of the sequential release of cytokines from PBMCs upon the activation of the immune response, with TNF- $\alpha$ being the first cytokine to be released by PBMCs, followed by IL-6 [36], which in turn promoted the differentiation of monocytes. Presently, LDPE ground particles induced a greater release of cytokines than the control (Fig. 7C and D). We did not observe release of IL-2 in response to PE treatment (Fig. S4).

PE is synthesized from ethylene gas in benzene using some catalysts, under conditions of high pressure and temperature. Therefore, PE is considered as one of the "safer plastics" compared to bisphenol A-containing plastics [37]. However, some additives, initiators, monomers, or impurities in PE might induce an inflammatory immune response. PE plastics can interact with macrophages (inflammatory response) [38], induce IL-6 production in osteoblasts [28, 29], and perpetuate chronic inflammation around PE implantation [39]. Additionally, in vivo implantation of $\mathrm{PE}$ can result in an accumulation of leukocytes, macrophages, and giant cells around the PE-implanted area, suggesting that PE plastics can cause an inflammatory reaction $[39,40]$. Hence, we concluded that the cellular responses are dependent on the morphology and concentration of PE. In particular, smooth HDPE particles do not cause serious toxicity but induce secretion of immune-related cytokines, and sharpedged ground LDPE particles at high concentrations cause both inflammation and cytotoxicity. We also believe that LDPE can more easily release additive chemicals because of its low crystallinity, resulting in a slight detrimental effect on cells. 


\subsection{Hemolysis of RBCs}

We further examined the effect of PE microplastics on the hemolysis of RBCs (Fig. 9). When the RBC membrane is damaged and ruptured physically or chemically, the cytoplasmic contents including hemoglobin are released, which can be detected by analyzing the absorbance of released hemoglobin. Unlike other assays, in this experiment, the particles and RBCs were incubated with agitation at $500 \mathrm{rpm}$, allowing the cells to directly contact the particles. A higher concentration of plastics induced greater hemolysis. We believe that the sharp edges or fragments of LDPE and HDPE particles (Fig. S1) and the slightly curved shape of HDPE compared with spheres (value $=0$, Fig. 4C) might physically rupture the RBC membrane, resulting in cell lysis.

\subsection{Histamine release and ROS production by PE microplastics treatment}

We also checked histamine release and ROS production induced by each particle. Histamine is one of the chemical mediators produced during an inflammatory response and is mainly secreted from basophils and mast cells [41]. Histamine release upon treatment with microparticles is shown in Fig. S5. The results indicated that smaller HDPE particles (1-10 $\mu \mathrm{m})$ tended to induce marginal histamine release. The observation that LPS did not induce histamine release is consistent with the findings of a previous report [42]. Examination of ROS production from HDFs upon treatment with LDPE (Fig. S6) indicated that high concentrations of LDPE microparticles could induce an environmental stress in normal cells.

We performed diverse in vitro assays to examine the effect of PE particles in human cells. It should be noted that although PE is non-lethal to human cells, it can exert chemical (remaining impurities in PE particles during synthesis) or physical (sharp edge of PE particles as a result of grinding and high concentration) effects on cells. Even though the PE 
microplastics did not have a serious effect on cells, we are still concerned that high concentrations of microplastics could induce cell death and potentially harm human cells upon continuous accumulation. Moreover, in a marine environment, the PE microplastics can adsorb bacteria, viruses, or toxic chemicals (persistent organic pollutants) onto their surface, thereby increasing the probability of a serious infection or disease when these particles are ingested by animals, including humans [8].

\section{Conclusion}

In this study, we have shown the effect of the physical aspects (morphology and concentration) of PE microplastics on human cells. PE is one of the most commonly used plastics and is mainly used in packaging in the form of plastic bags, containers, plastic films (bulk plastics), and scrubs in cosmetics (microplastics). To investigate the toxicity of PE microplastics in vitro, we examined the effect of microbeads and ground bulk plastics on cultured cells. The two types of microplastics used included 1-100 $\mu \mathrm{m}$-sized HDPE particles and milled microdebris from bulk LDPE pellets (size range, 25-75 $\mu \mathrm{m}$ and $75-200 \mu \mathrm{m}$ ). We statistically quantified the ruggedness of the microplastics from SEM images of both microplastics to correlate with the results of toxicological experiments. The analysis indicated a significant (10 times greater) curvature value of LDPE microparticles, which means more randomness with respect to their shape. In the toxicological experiments, HDPE particles did not cause significant cytotoxicity in cells because of their relatively smooth surface morphology, but induced an immune response in PBMCs and enhanced PBMC differentiation. In addition, high concentrations of HDPE particles resulted in hemolysis of RBCs when samples were agitated during incubation. Ground LDPE particles caused 
increased cytotoxicity, inflammatory response, hemolysis, and ROS production at high concentrations, probably because of the sharp edges of the particles (higher curvature change) and smaller pieces derived from microdebris. PE is recognized as a biologically safe material.

Our results indicated that the PE microplastics did not show significant cytotoxicity. However, we suggest that there are possibilities of side effects, such as cytotoxicity and induction of inflammatory response, after exposure to microplastics with specific concentrations or shapes. PE microdebris in marine environments can easily absorb bacteria, viruses, or toxic chemicals present in the ocean, and when accumulated, might cause damage to the marine biota and humans.

\section{Ethics approval and consent to participate}

Not applicable

\section{Consent for publication}

467 All authors consent to the publication of this manuscript.

\section{Availability of data and materials}

All data generated or analyzed during this study are included in this published article and its supplementary information files.

\section{Competing interests}

474 The authors declare that there are no conflicts of interest. 
$476 \quad$ Funding

477 The authors confirm that there are no known conflicts of interest associated with this 478 publication. This research was supported by Basic Science Research Program through the 479 National Research Foundation of Korea (NRF) funded by the Ministry of Science and ICT $480 \quad$ (NRF-2017R1E1A1A01074343).

481

482 Authors' contributions

483 D. Choi performed most of the toxicology assays and prepared the manuscript. J. Hwang and 484 S. Han carried out the toxicology assays. J. Bang and Y. Hwang determined the statistical 485 shape analysis of PE particles. T. Kim and Y. Oh prepared spherical and ground PE particles. 486 J. Choi and J. Hong supervised the project and examined the final version of the manuscript. 487

Acknowledgements

489 Not applicable

490

$491 \quad$ Author information

492 Not applicable 
1. Hidalgo-Ruz V, Gutow L, Thompson RC, Thiel M. Microplastics in the marine environment: a review of the methods used for identification and quantification. Environ Sci Technol. 2012;46 6:3060-75.

2. Martin J, Lusher A, Thompson RC, Morley A. The deposition and accumulation of microplastics in marine sediments and bottom water from the Irish continental shelf. Sci Rep. 2017;7 1:10772.

3. Pettipas S, Bernier M, Walker TR. A Canadian policy framework to mitigate plastic marine pollution. Mar Policy. 2016;68:117-22.

4. Kontrick AV: Microplastics and human health: our great future to think about now. Springer; 2018.

5. Wessel CC, Lockridge GR, Battiste D, Cebrian J. Abundance and characteristics of microplastics in beach sediments: insights into microplastic accumulation in northern Gulf of Mexico estuaries. Mar Pollut Bull. 2016;109 1:178-83.

6. Courtene-Jones W, Quinn B, Gary SF, Mogg AO, Narayanaswamy BE. Microplastic pollution identified in deep-sea water and ingested by benthic invertebrates in the Rockall Trough, North Atlantic Ocean. Environ Pollut. 2017;231:271-80.

7. Woodall LC, Sanchez-Vidal A, Canals M, Paterson GL, Coppock R, Sleight V, et al. The deep sea is a major sink for microplastic debris. Royal Soc Open Sci. 2014;1 $4: 140317$.

8. Law KL, Thompson RC. Microplastics in the seas. Science. 2014;345 6193:144-5.

9. Feng Z, Zhang T, Li Y, He X, Wang R, Xu J, et al. The accumulation of microplastics in fish from an important fish farm and mariculture area, Haizhou Bay, China. Science of The Total Environment. 2019;696:133948. 
10. Yang H, Liu C, Yang D, Zhang H, Xi Z. Comparative study of cytotoxicity, oxidative stress and genotoxicity induced by four typical nanomaterials: the role of particle size, shape and composition. J Appl Toxicol. 2009;29 1:69-78.

11. Yamamoto A, Honma R, Sumita M, Hanawa T. Cytotoxicity evaluation of ceramic particles of different sizes and shapes. Journal of Biomedical Materials Research Part A. $2004 ; 68$ 2:244-56.

12. Lee YJ, Ahn E-Y, Park Y. Shape-dependent cytotoxicity and cellular uptake of gold nanoparticles synthesized using green tea extract. Nanoscale Res Lett. 2019;14 1:129.

13. Liu S, Wei L, Hao L, Fang N, Chang MW, Xu R, et al. Sharper and faster "nano darts" kill more bacteria: a study of antibacterial activity of individually dispersed pristine single-walled carbon nanotube. ACS nano. 2009;3 12:3891-902.

14. Lu Y, Zhang Y, Deng Y, Jiang W, Zhao Y, Geng J, et al. Uptake and accumulation of polystyrene microplastics in zebrafish (Danio rerio) and toxic effects in liver. Environ Sci Technol. 2016;50 7:4054-60.

15. Lee K-W, Shim WJ, Kwon OY, Kang J-H. Size-dependent effects of micro polystyrene particles in the marine copepod Tigriopus japonicus. Environ Sci Technol. 2013;47 19:11278-83.

16. Zhang C, Chen X, Wang J, Tan L. Toxic effects of microplastic on marine microalgae Skeletonema costatum: interactions between microplastic and algae. Environ Pollut. 2017;220:1282-8.

17. Ma Y, Huang A, Cao S, Sun F, Wang L, Guo H, et al. Effects of nanoplastics and microplastics on toxicity, bioaccumulation, and environmental fate of phenanthrene in fresh water. Environ Pollut. 2016;219:166-73.

18. Collard F, Gilbert B, Eppe G, Parmentier E, Das K. Detection of anthropogenic 
particles in fish stomachs: an isolation method adapted to identification by Raman spectroscopy. Archives of environmental contamination and toxicology. 2015;69 3:331-9.

19. Enders K, Lenz R, Stedmon CA, Nielsen TG. Abundance, size and polymer composition of marine microplastics $\geq 10 \mu \mathrm{m}$ in the Atlantic Ocean and their modelled vertical distribution. Mar Pollut Bull. 2015;100 1:70-81.

20. Furukawa T, Sato H, Kita Y, Matsukawa K, Yamaguchi H, Ochiai S, et al. Molecular structure, crystallinity and morphology of polyethylene/polypropylene blends studied by Raman mapping, scanning electron microscopy, wide angle X-ray diffraction, and differential scanning calorimetry. Polym J. 2006;38 11:1127.

21. Bartczak Z, Argon A, Cohen R, Weinberg M. Toughness mechanism in semicrystalline polymer blends: I. High-density polyethylene toughened with rubbers. Polymer. 1999;40 9:2331-46.

22. Zhang Y, Li Z, Li H, Gao J, Zhang J, Zeng Y. Effect of carbon nanotubes shape on the properties of multiwall carbon nanotubes/polyethylene flexible transparent conductive films. J Mater Sci: Mater Electron. 2014;25 6:2692-6.

23. Kitao K. A study of brittle-ductile transition in polyethylene. Polym Eng Sci. 1997;37 5:777-88.

24. Cox KD, Covernton GA, Davies HL, Dower JF, Juanes F, Dudas SE. Human Consumption of Microplastics. Environ Sci Technol. 2019;53 12:7068-74.

25. Galloway TS. Micro-and nano-plastics and human health. Marine anthropogenic litter: Springer, Cham; 2015. p. 343-66.

26. Monnery BD, Wright M, Cavill R, Hoogenboom R, Shaunak S, Steinke JH, et al. Cytotoxicity of polycations: relationship of molecular weight and the hydrolytic 
theory of the mechanism of toxicity. Int J Pharm. 2017;521 1-2:249-58.

27. Xia T, Kovochich M, Liong M, Zink JI, Nel AE. Cationic polystyrene nanosphere toxicity depends on cell-specific endocytic and mitochondrial injury pathways. ACS nano. 2007;2 1:85-96.

28. Rodrigo A, Martínez M, Escudero M, Ruíz J, Martínez P, Saldana L, et al. Influence of particle size in the effect of polyethylene on human osteoblastic cells. Biomaterials. $2001 ; 228: 755-62$

29. Rodrigo A, Martínez M, Saldana L, Valles G, Martínez P, González-Carrasco JL, et al. Effects of polyethylene and $\alpha$-alumina particles on IL- 6 expression and secretion in primary cultures of human osteoblastic cells. Biomaterials. 2002;23 3:901-8.

30. Tanaka T, Narazaki M, Kishimoto T. IL-6 in inflammation, immunity, and disease. CSH Perspect Biol. 2014;6 10:a016295.

31. Castell JV, Gómez-Lechón MJ, David M, Andus T, Geiger T, Trullenque R, et al. Interleukin- 6 is the major regulator of acute phase protein synthesis in adult human hepatocytes. FEBS Lett. 1989;242 2:237-9.

32. Pinto LM, Oliveira SA, Braga EL, Nogueira RM, Kubelka CF. Increased proinflammatory cytokines (TNF-a and IL-6) and anti-inflammatory compounds (sTNFRp55 and sTNFRp75) in Brazilian patients during exanthematic dengue fever. Mem Inst Oswaldo Cruz. 1999;94 3:387-94.

33. Claesson-Welsh L. Vascular permeability-the essentials. Ups J Med Sci Suppl. 2015;120 3:135-43.

34. Popa C, Netea MG, Van Riel PL, Van Der Meer JW, Stalenhoef AF. The role of TNF$\alpha$ in chronic inflammatory conditions, intermediary metabolism, and cardiovascular risk. J Lipid Res. 2007;48 4:751-62. 
35. Duddy M, Dickson G, Hawkins S, Armstrong M. Monocyte-derived dendritic cells: a potential target for therapy inmultiple sclerosis (MS). Clin Exp Immunol. 2001;123 2:280-7.

36. Jansky L, Reymanova P, Kopecky J. Dynamics of cytokine production in human peripheral blood mononuclear cells stimulated by LPS, or infected by Borrelia. Physioll Res. 2003;52 5:593-8.

37. Sandler S. Polymer syntheses. vol. 1: Elsevier; 2012.

38. Xing S, Santerre J, Labow R, Boynton E. The effect of polyethylene particle phagocytosis on the viability of mature human macrophages. Journal of Biomedical Materials Research: An Official Journal of The Society for Biomaterials, The Japanese Society for Biomaterials, and The Australian Society for Biomaterials and the Korean Society for Biomaterials. 2002;61 4:619-27.

39. Xing S, Santerre J, Labow R, Boynton E. The effect of polyethylene particle phagocytosis on the viability of mature human macrophages. Journal of Biomedical Materials Research Part A. 2002;61 4:619-27.

40. Bing J. The tissue reaction to implanted plastics. APMIS. 1955;105:16-26.

41. Borriello F, Iannone R, Marone G. Histamine release from mast cells and basophils. Histamine and Histamine Receptors in Health and Disease: Springer; 2017. p. 121-39.

42. Watanabe Y, Todome Y, Ohkuni H, Sakurada S, Ishikawa T, Yutsudo T, et al. Cysteine protease activity and histamine release from the human mast cell line HMC-1 stimulated by recombinant streptococcal pyrogenic exotoxin B/streptococcal cysteine protease. Infect Immun. 2002;70 7:3944-7. 


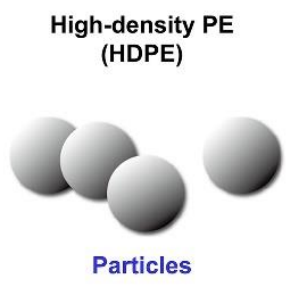

Density: $0.960 \mathrm{~g} / \mathrm{cc}$ Shape: Spherical Size: $1-10 \mu \mathrm{m} / 50 \mu \mathrm{m} / 100 \mu \mathrm{m}$
Polyethylene (PE)<smiles>CCCCC(C)(C)C</smiles>

Low-density PE (LDPE)

\section{Statistical shape analysis}

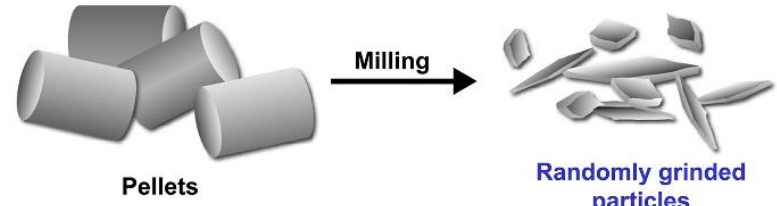

Density: $0.925 \mathrm{~g} / \mathrm{cc}$

particles

Shape: Random

Size: $25-75 \mu \mathrm{m} / 75-200 \mu \mathrm{m}$

\section{Toxicology analysis}

\section{Curvature differences}
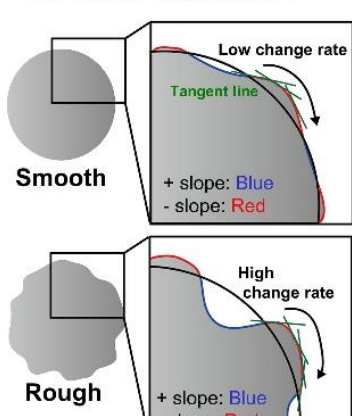
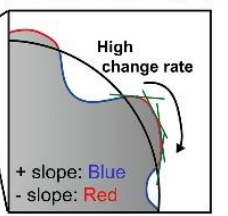

Quadratic function of curvature

$$
y=a x^{2}+b x+c
$$
Tangent line (slope) $y^{\prime}=2 a x+b$

Change rate of curvature $\downarrow$

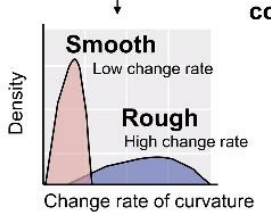

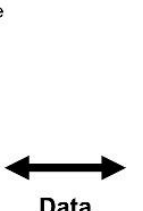

Data

correlation 3. HDF/ HeLa/ RBL-2H3

- Viability (CCk-8)



- Viability

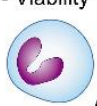

Live

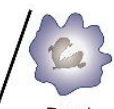

- Cytokine release - Differentiation

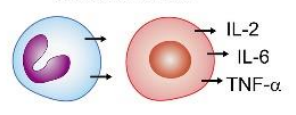

2. HMC-1

- Histamine release

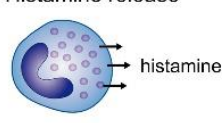

4. RBC

- Hemolysis test

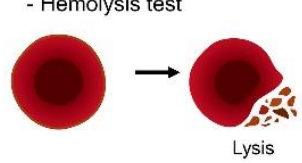

614 Fig. 1. Schematic illustration of HDPE and LDPE particles and toxicity analysis in terms of

615 the physical aspects of PE microplastics by correlating statistical data and in vitro toxicity

616 results. PBMC: peripheral blood mononuclear cells; HMC-1: human mast cell-1; HDF:

617 human dermal fibroblast; HeLa: cervical cancer cells; RBL-2H3: basophilic leukemia cells;

618 RBC: red blood cell; CCK-8: cell counting kit-8; WST-8: water-soluble tetrazolium salt-8. 
A
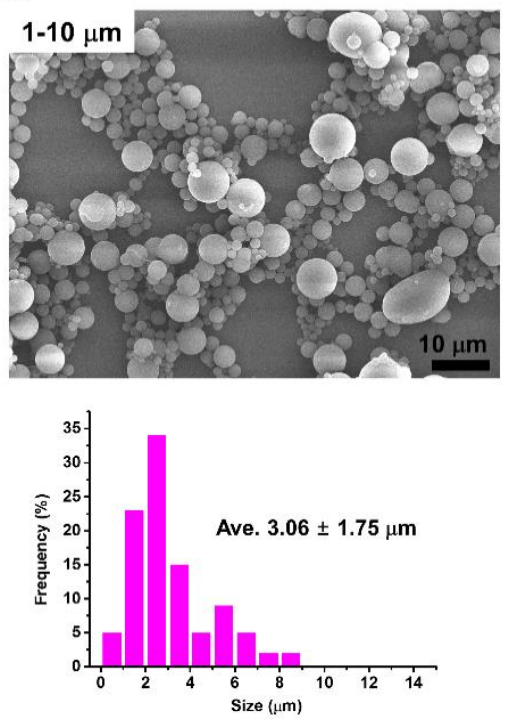

B
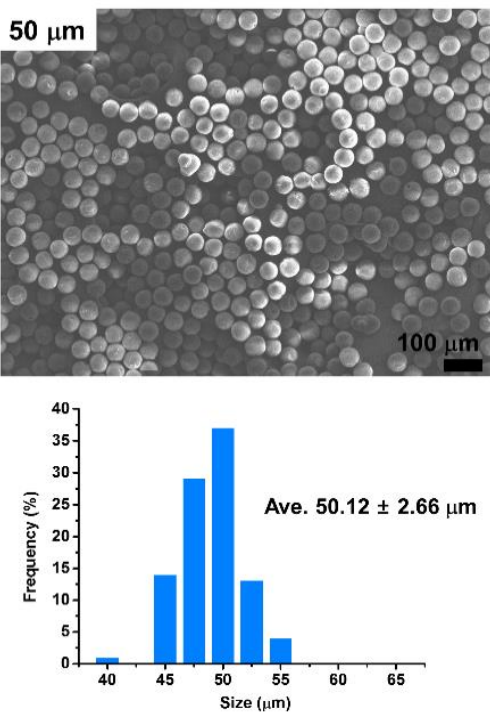

C
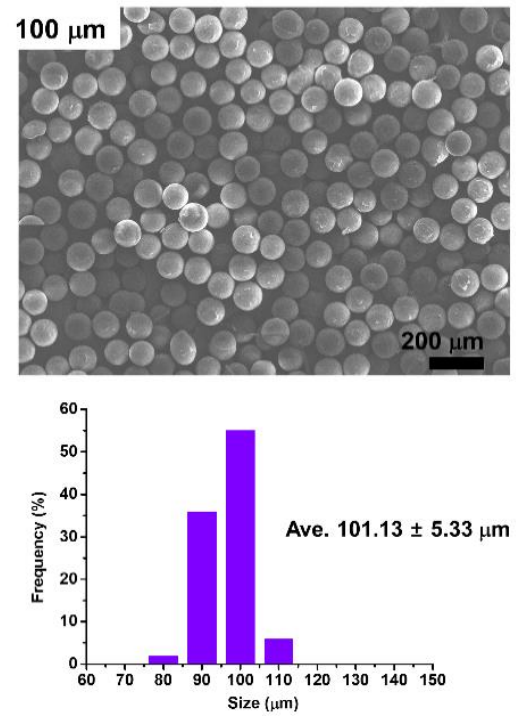

Fig. 2. Representative images and overall size distribution of (A) 1-10 $\mu \mathrm{m}$, (B) $50 \mu \mathrm{m}$, and

(C) $100 \mu \mathrm{m}$ HDPE particles $(\mathrm{n}=100)$ calculated from SEM images. Ave: average size.

A

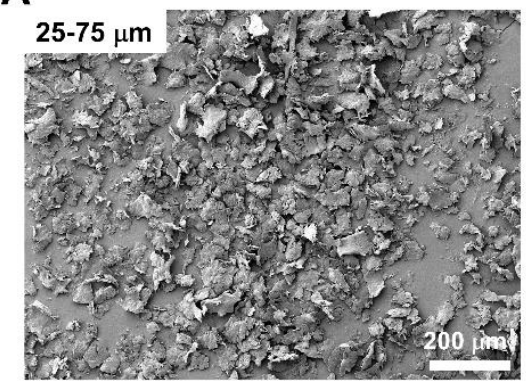

B



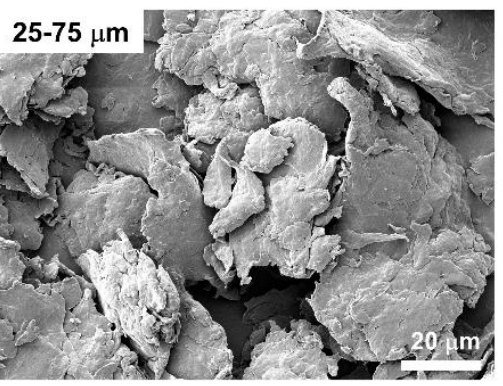

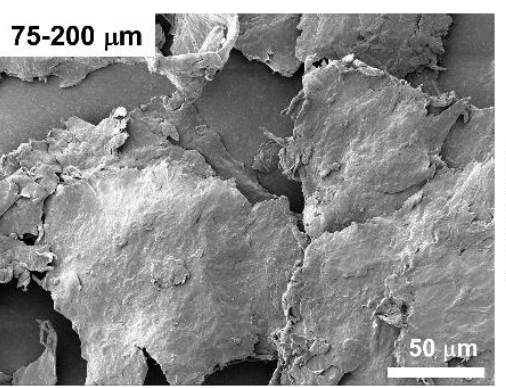





Fig. 3. Representative image and overall area distribution of (A) $25-75 \mu \mathrm{m}$ and (B) $75-200$ $\mu \mathrm{m}$ LDPE microparticles $(\mathrm{n}=50)$ calculated from SEM images. Ave: average area. 
A
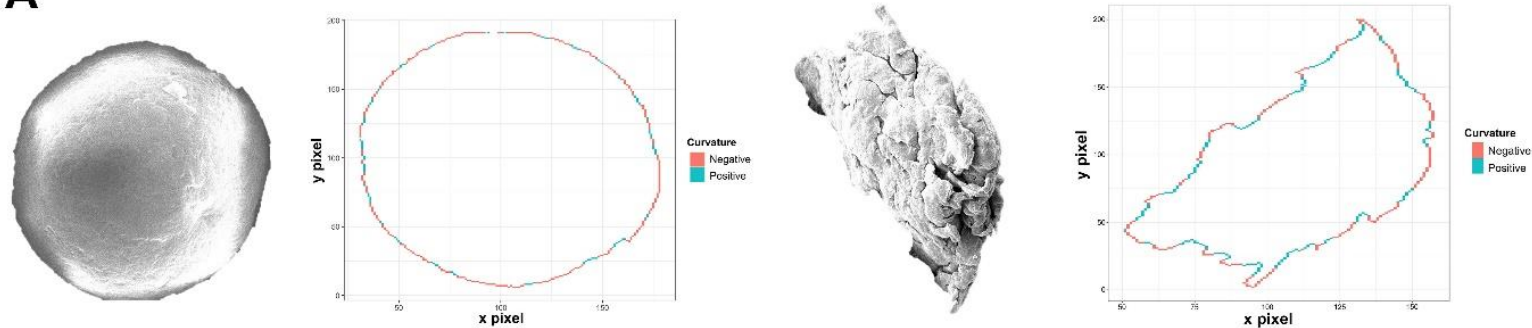

B



C

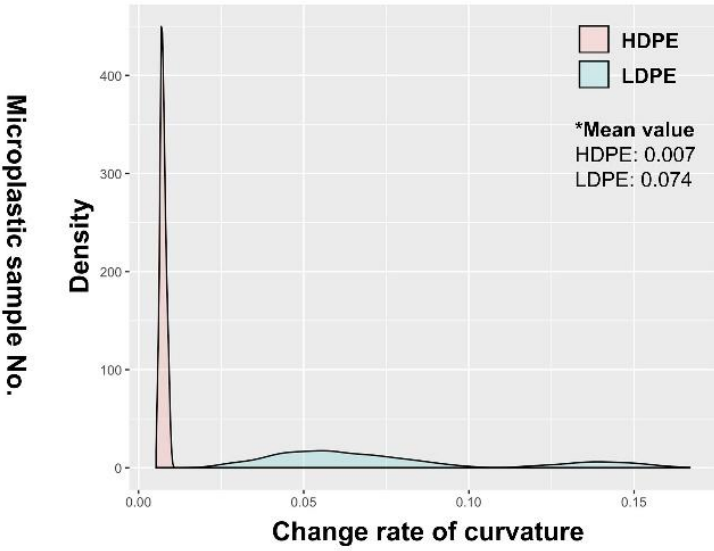

Fig. 4. Local curvature of LDPE and HDPE particles. (A) Background-removed SEM image

of HDPE and LDPE particles and their local curvature and (B) change rate of the curvature as

a function of boundary pixel of each microplastic $(n=4)$. (C) Average distribution of curvature variation. 
A

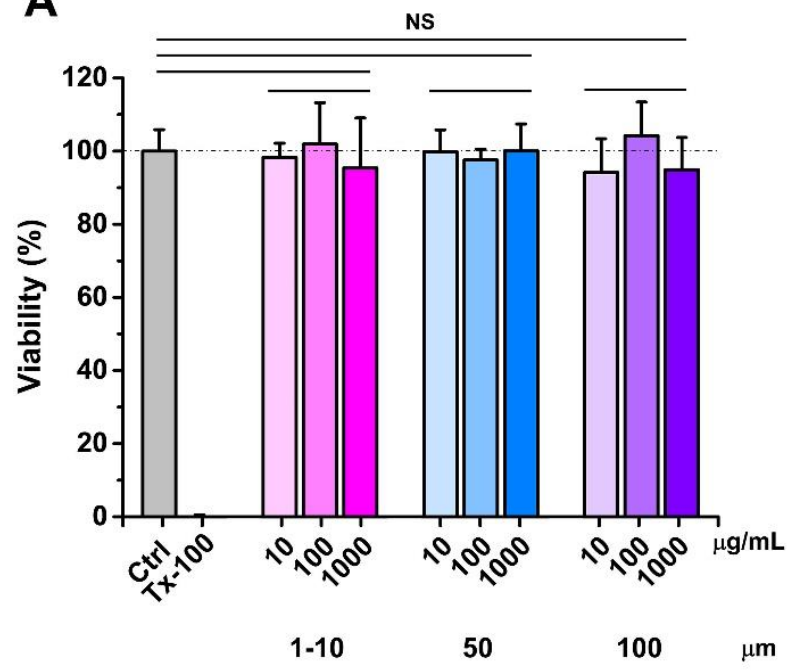

B

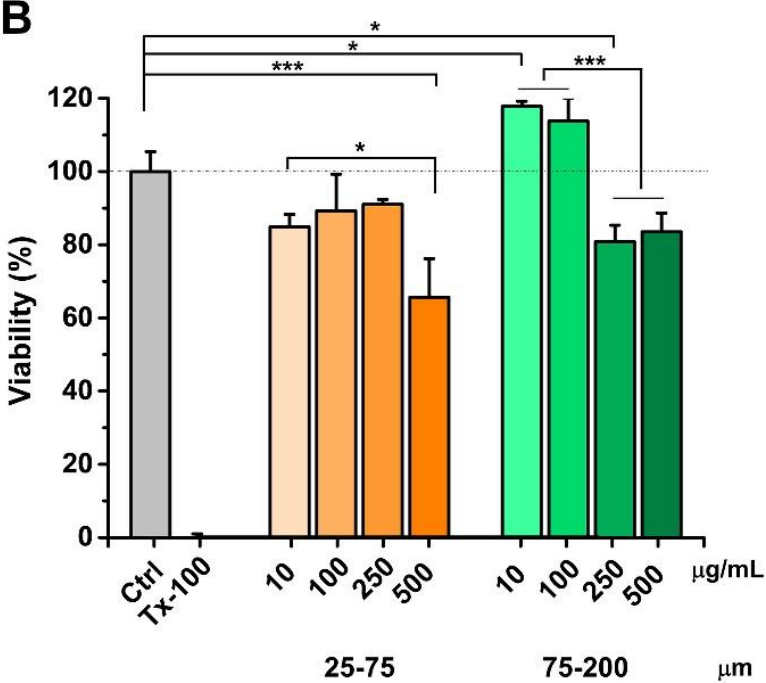

635 Fig. 5. Live/dead ratio of PBMCs upon treatment with (A) HDPE microparticles and (B)

636 LDPE ground particles for $24 \mathrm{~h}$. Statistical analysis was performed by ANOVA followed by

637 Tukey's post hoc test $(\mathrm{n}=3)$. NS and * indicate "non-significant" and " $p<0.05$ " compared to $\mathrm{Ctrl}$ (Control), respectively. 

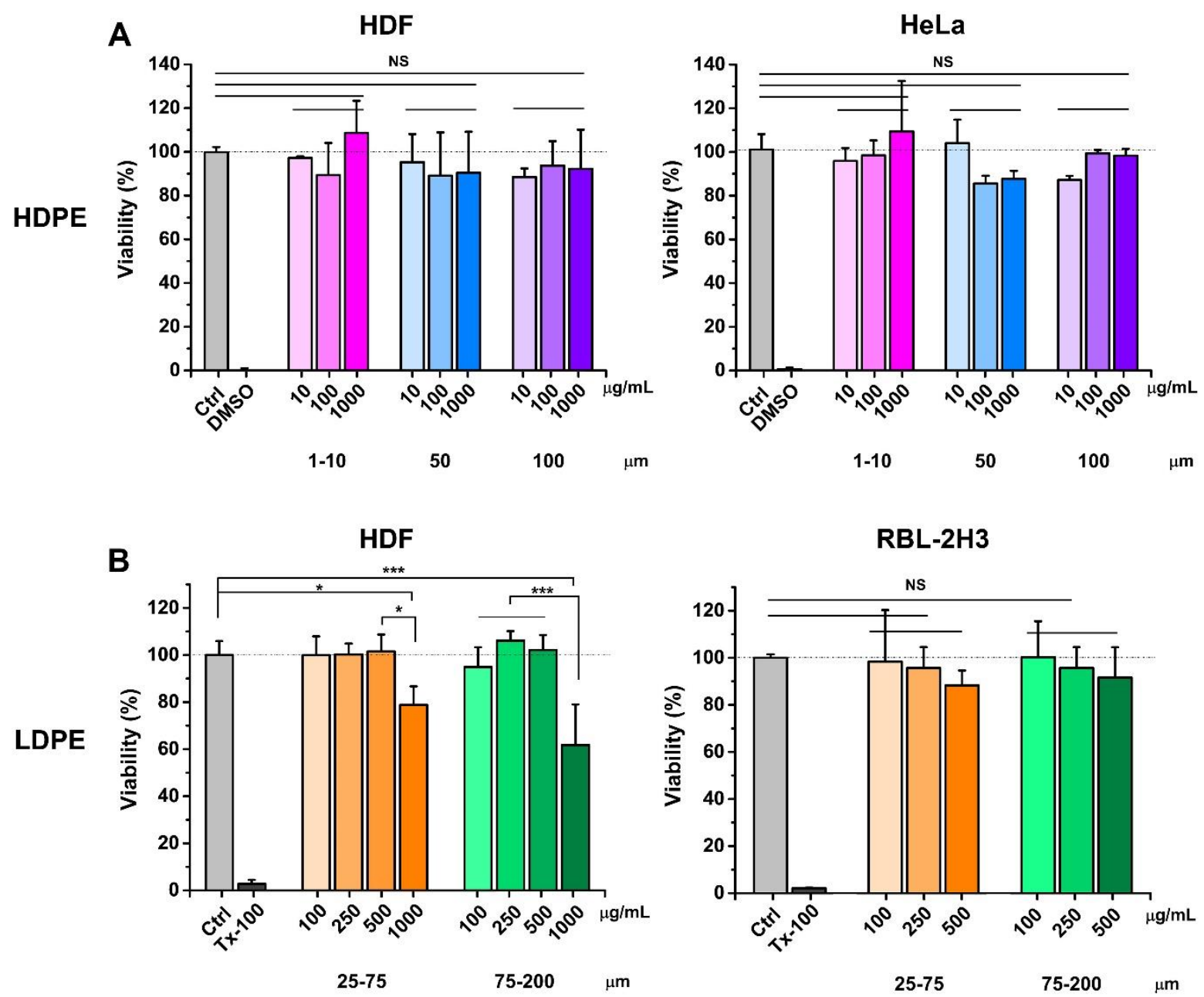

Fig. 6. Viability of (A) HDF and HeLa cells in response to HDPE particles, and that of (B)

642 HDF and RBL-2H3 cells in response to LDPE particles. Statistical analysis was performed by

ANOVA followed by Tukey's post hoc test $(\mathrm{n}=3)$. NS and * indicate "non-significant" and " $p<0.05 "$ compared to $\mathrm{Ctrl}$ (Control), respectively. 

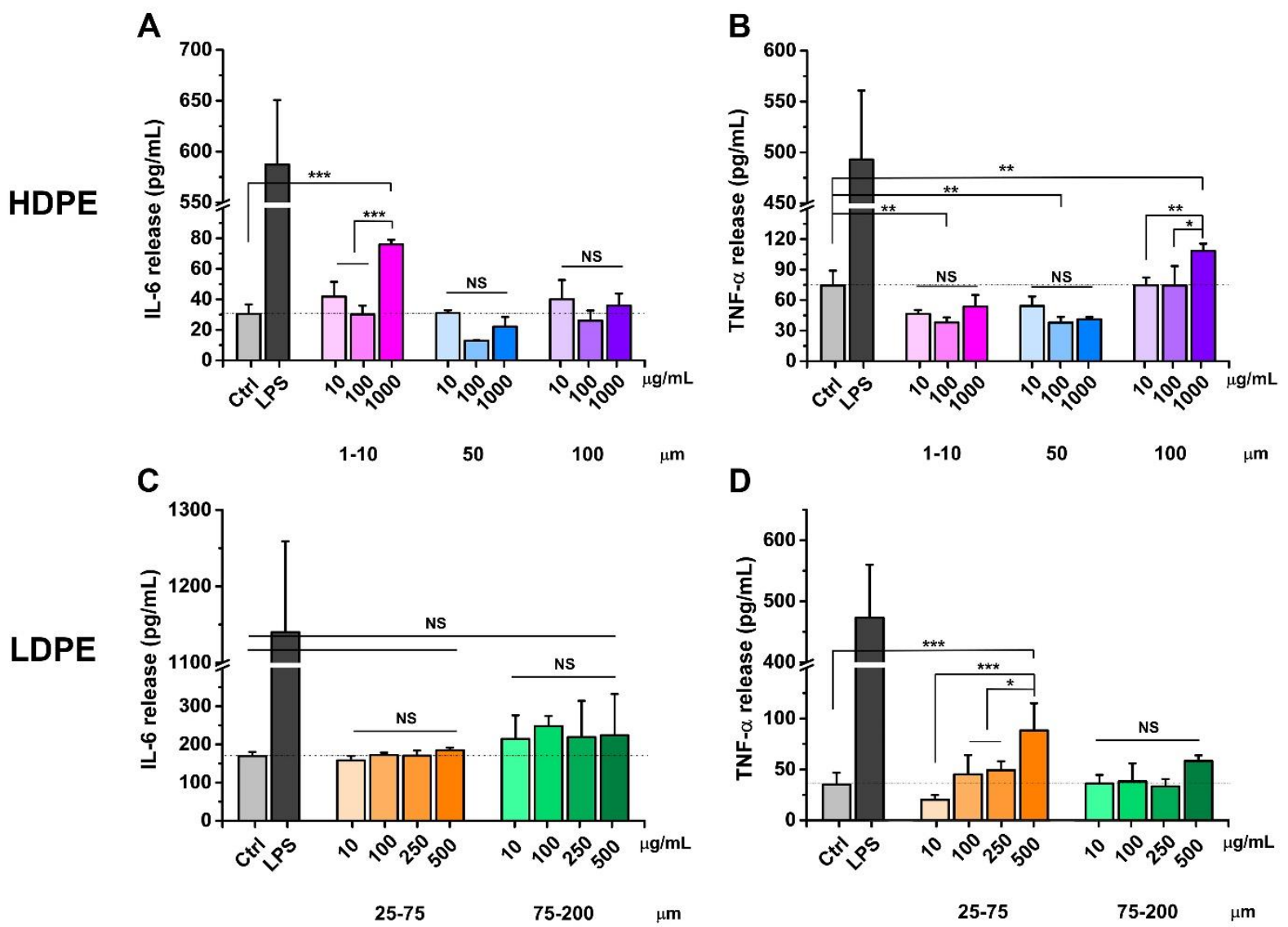

Fig. 7. Cytokine (IL-6 and TNF-a) release from PBMCs after four days of culture with HDPE 649 and LDPE particles at different concentrations. LPS $(5 \mu \mathrm{g} / \mathrm{mL})$ treated cells were used as a 650 positive control. Statistical analysis was performed by ANOVA followed by Tukey's post hoc 651 test $(\mathrm{n}=3)$. NS, *,**, and *** indicate "non-significant", " $p<0.05$," " $p<0.01$," and " $p<$ $0.001, "$ respectively. 


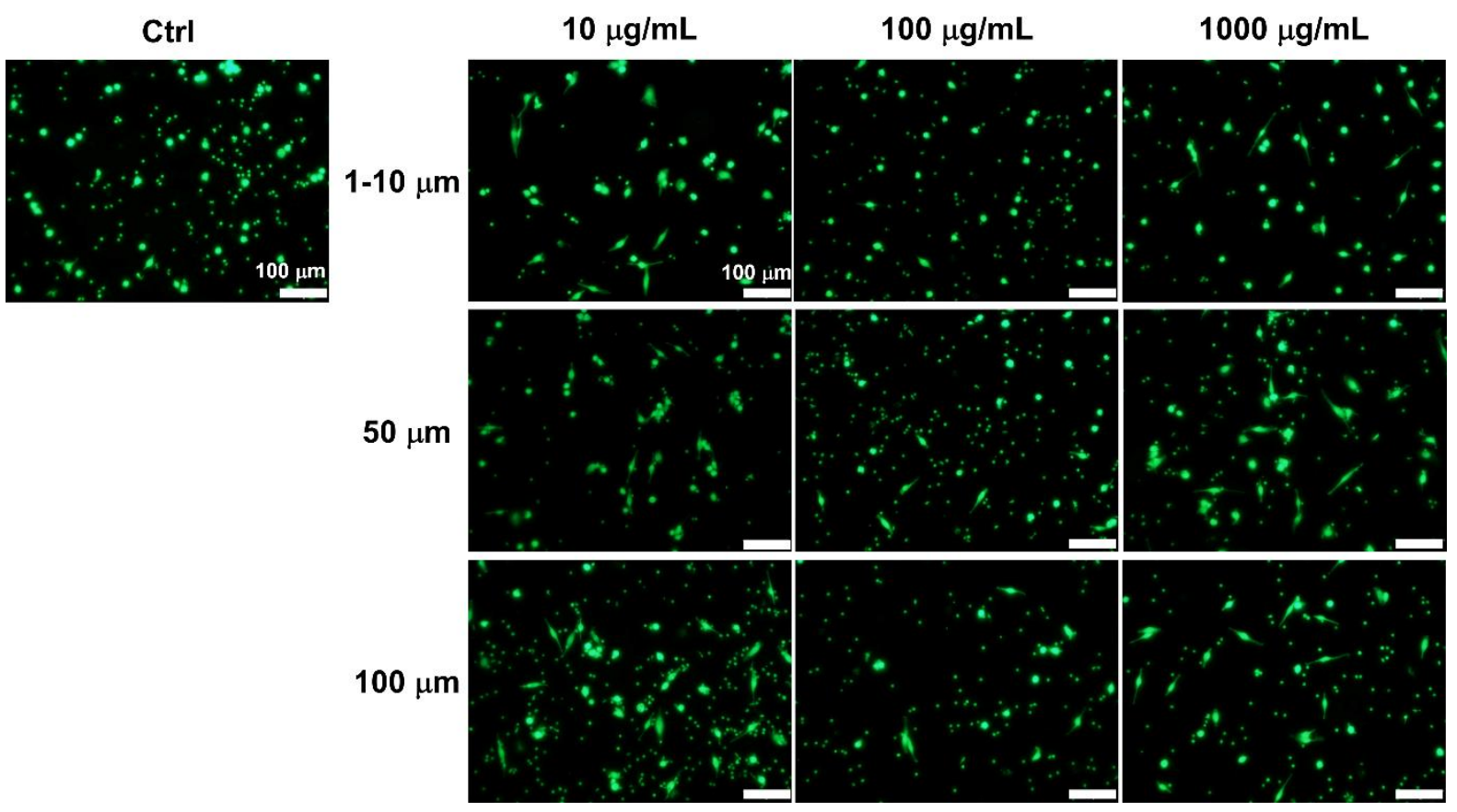

Fig. 8. Fluorescence images of PBMCs after five days of treatment with HDPE particles. 656 Live cells were stained by Calcein AM. The scale bar represents $100 \mu \mathrm{m}$.

A

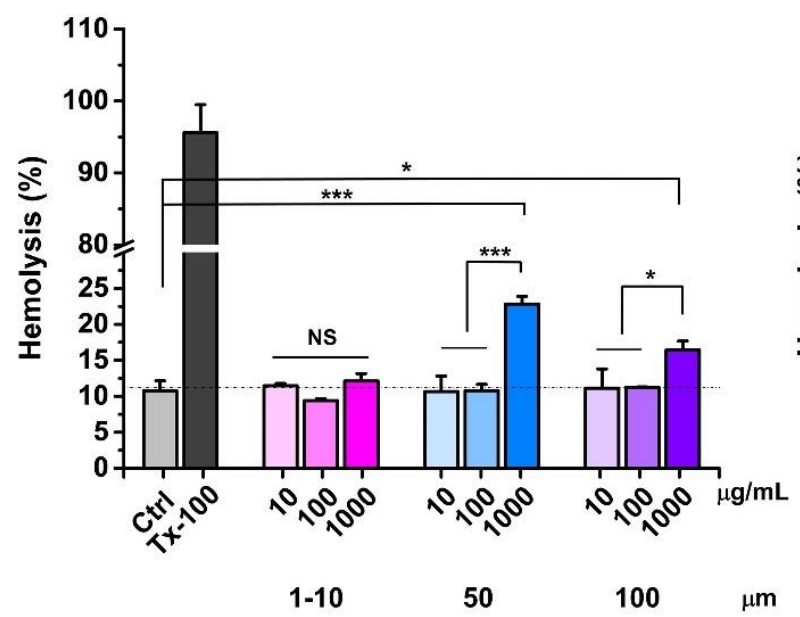

B

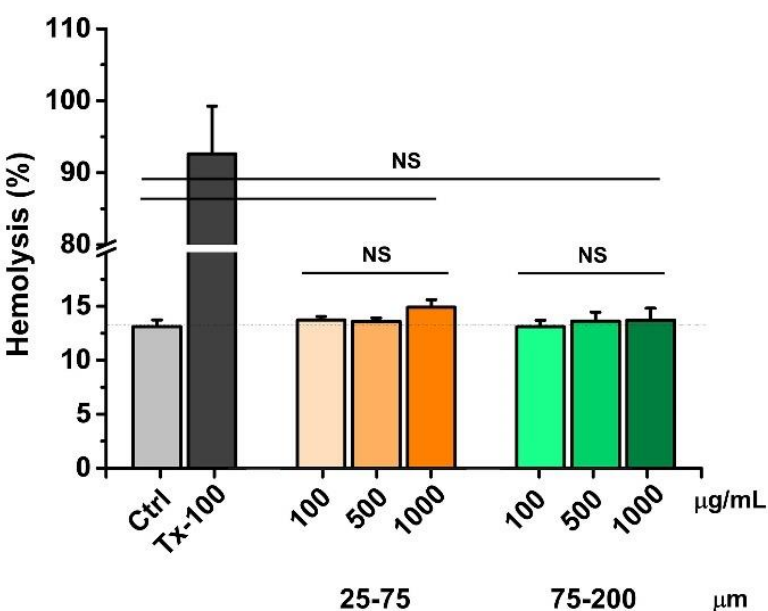

Fig. 9. Cytotoxicity of PE particles toward RBCs. Hemolysis ratio in RBCs upon exposure to

(A) HDPE and (B) LDPE particles for $1 \mathrm{~h}$. Statistical analysis was performed by ANOVA

661 followed by Tukey's post-hoc test $(\mathrm{n}=3)$. *, **, and *** indicate " $p<0.05$, , " $p<0.01$," and 
$662 " p<0.001$," respectively. 


\section{Figures}

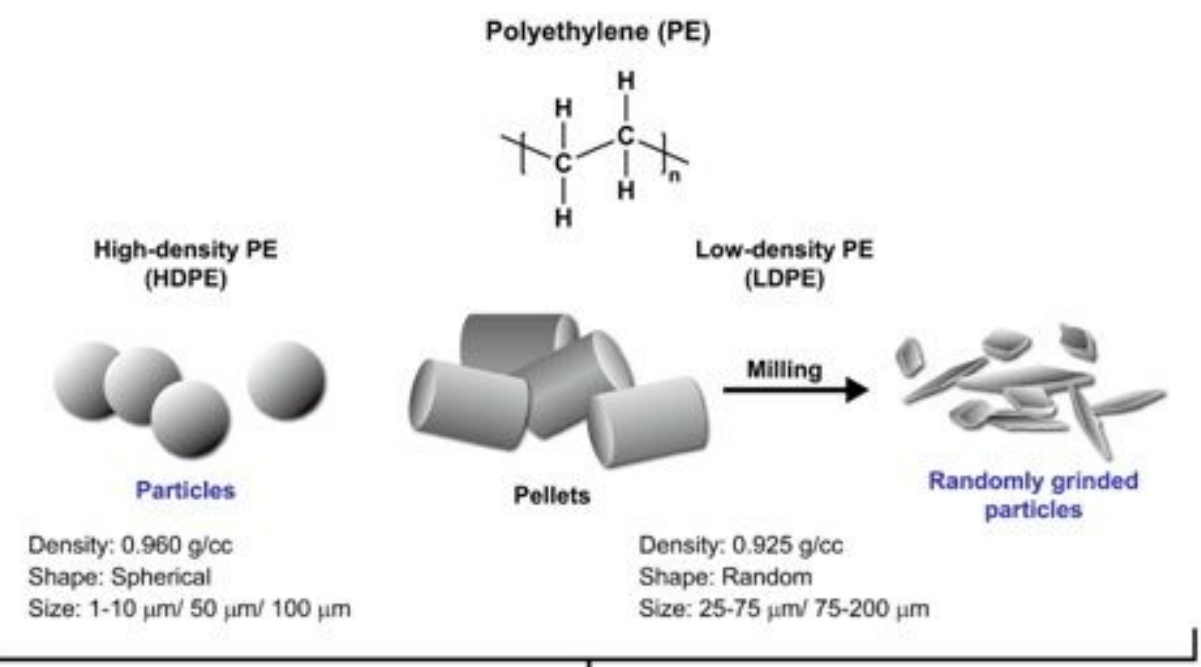

1. Statistical shape analysis

Curvature differences

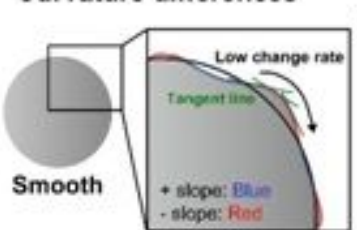

Quadratic function of curvature
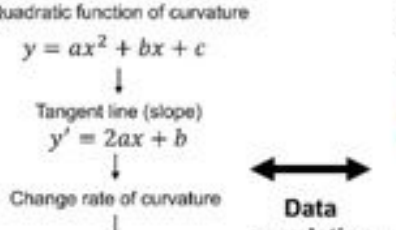

1. PBMC

2. Toxicology analysis
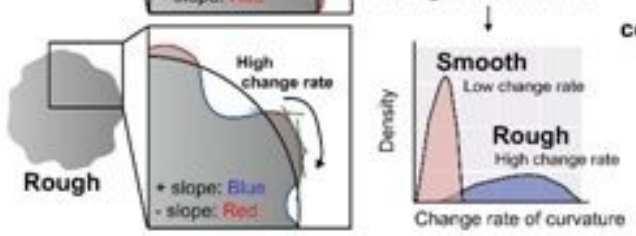

correlation

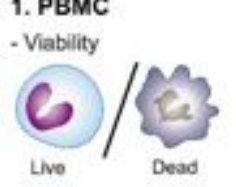

- Cytokine relcase

- Differentiation

2. HMC-1

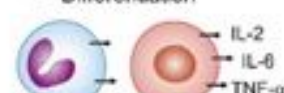

- Histamine release

3. HDF/ HeLa/ RBL-2H3

4. RBC

- Viability (CCK-8)
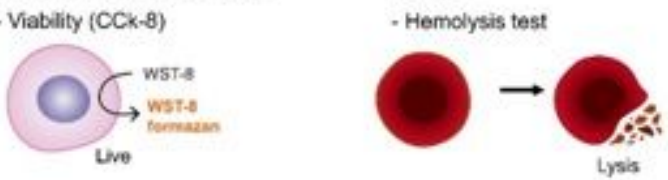

\section{Figure 1}

Schematic illustration of HDPE and LDPE particles and toxicity analysis in terms of the physical aspects of PE microplastics by correlating statistical data and in vitro toxicity results. PBMC: peripheral blood mononuclear cells; HMC-1: human mast cell-1; HDF: human dermal fibroblast; HeLa: cervical cancer cells; RBL-2H3: basophilic leukemia cells; RBC: red blood cell; CCK-8: cell counting kit-8; WST-8: water-soluble tetrazolium salt-8. 
A
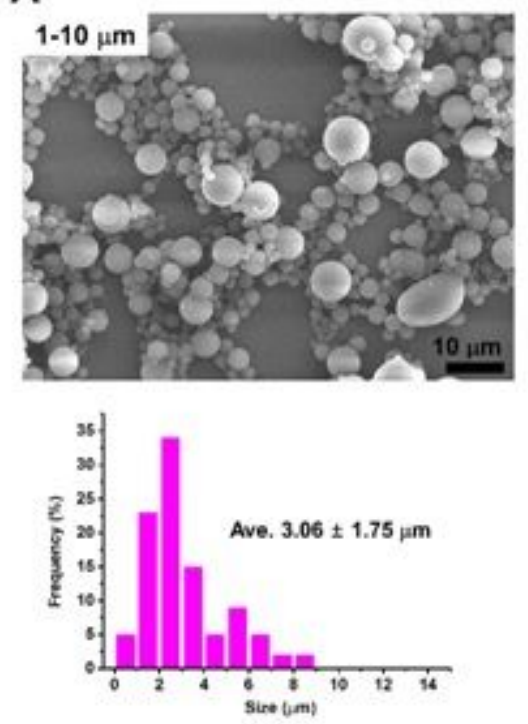

B
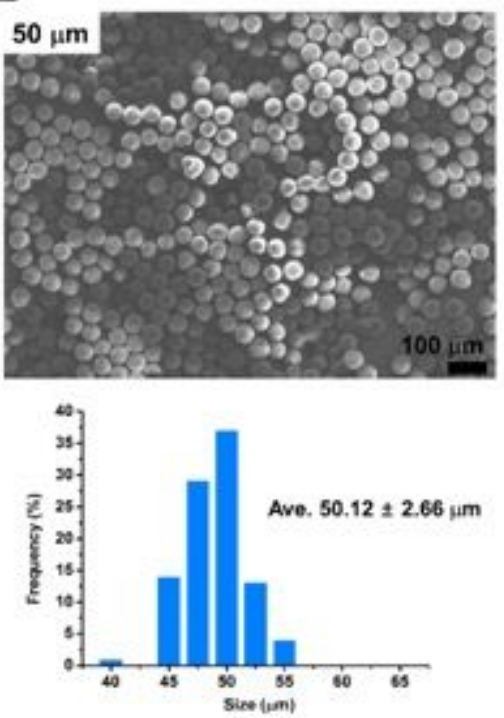

C
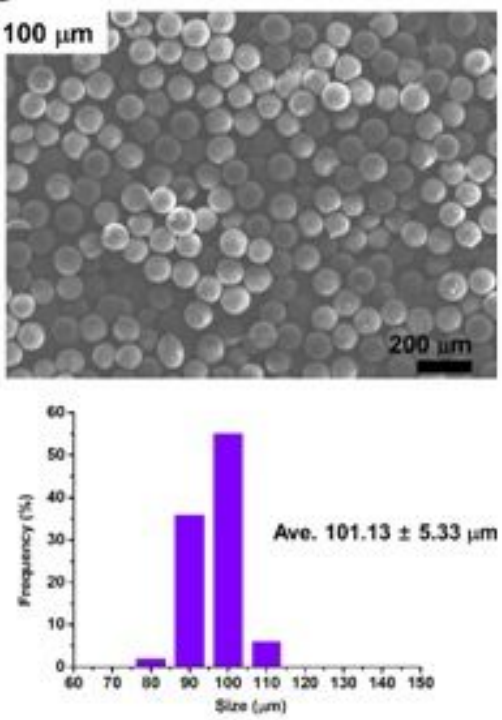

Figure 2

Representative images and overall size distribution of (A) 1-10 Im, (B) $50 \mathrm{Dm}$, and (C) $100 \mathrm{Dm}$ HDPE particles $(n=100)$ calculated from SEM images. Ave: average size.

A
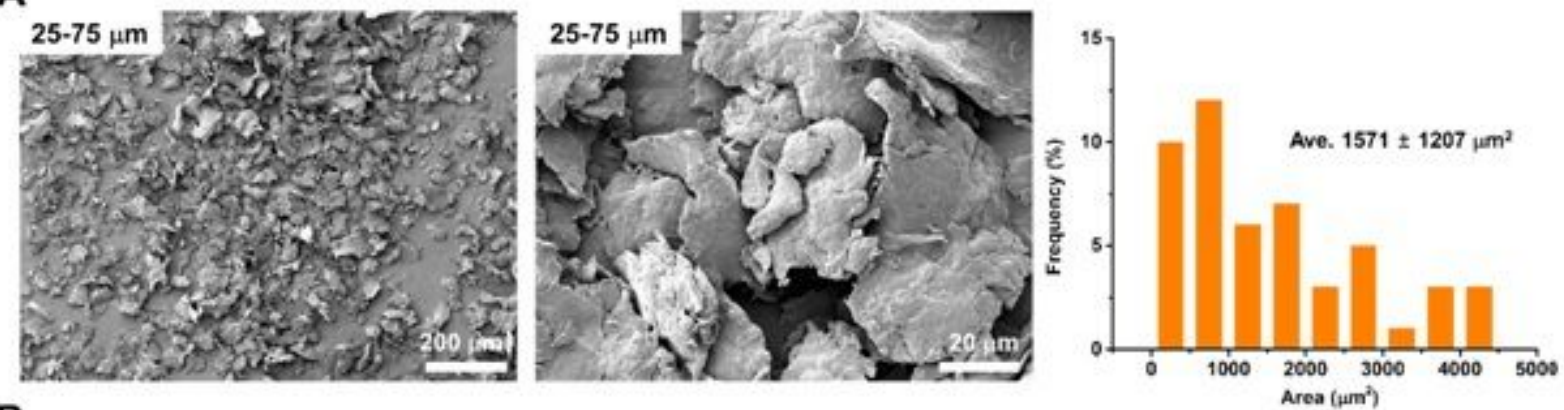

B
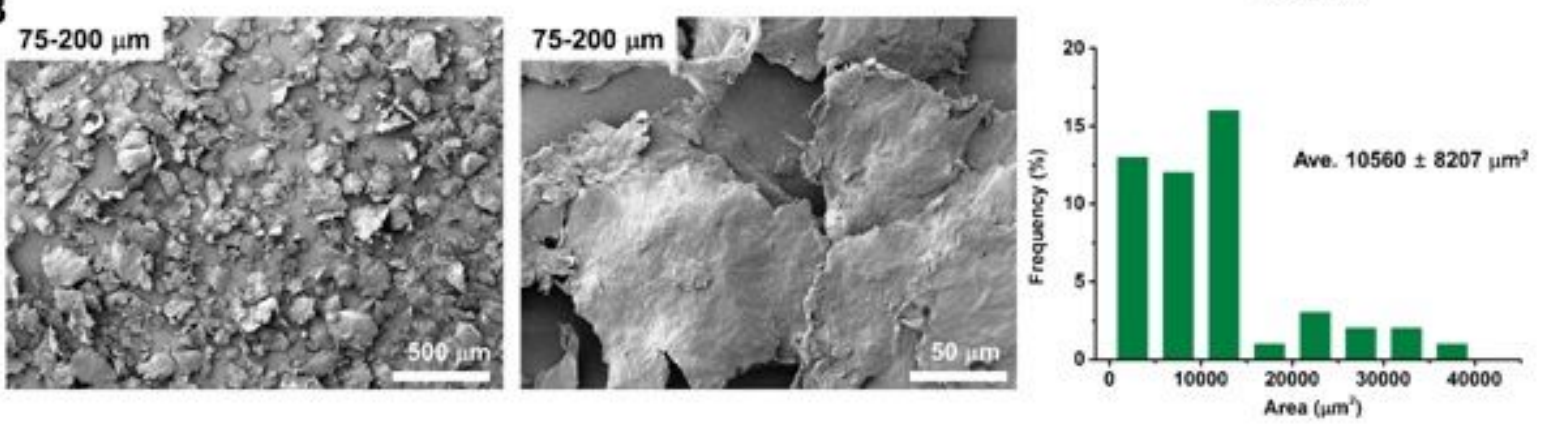

Figure 3

Representative image and overall area distribution of (A) 25-75 $\mathrm{mm}$ and (B) 75-200 Dm LDPE microparticles $(n=50)$ calculated from SEM images. Ave: average area. 
A
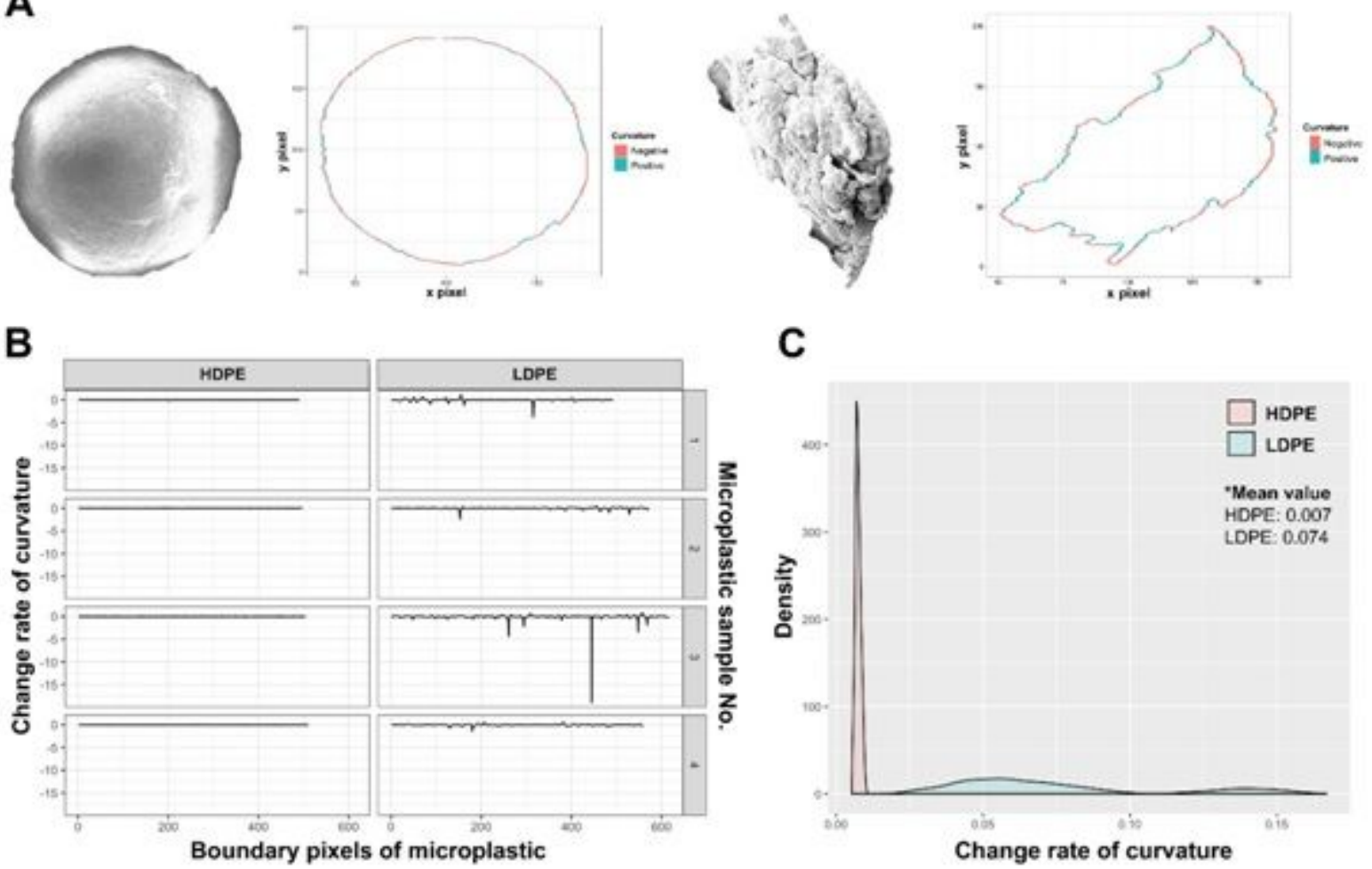

C

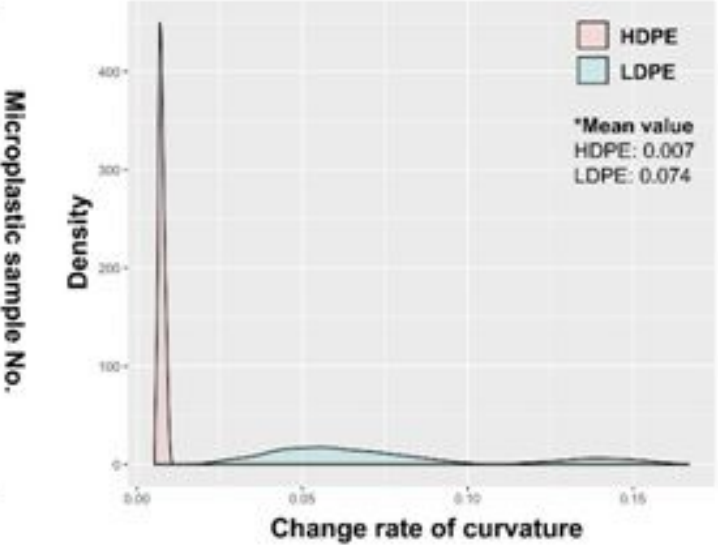

Figure 4

Local curvature of LDPE and HDPE particles. (A) Background-removed SEM image of HDPE and LDPE particles and their local curvature and $(B)$ change rate of the curvature as a function of boundary pixel of each microplastic $(n=4)$. (C) Average distribution of curvature variation.
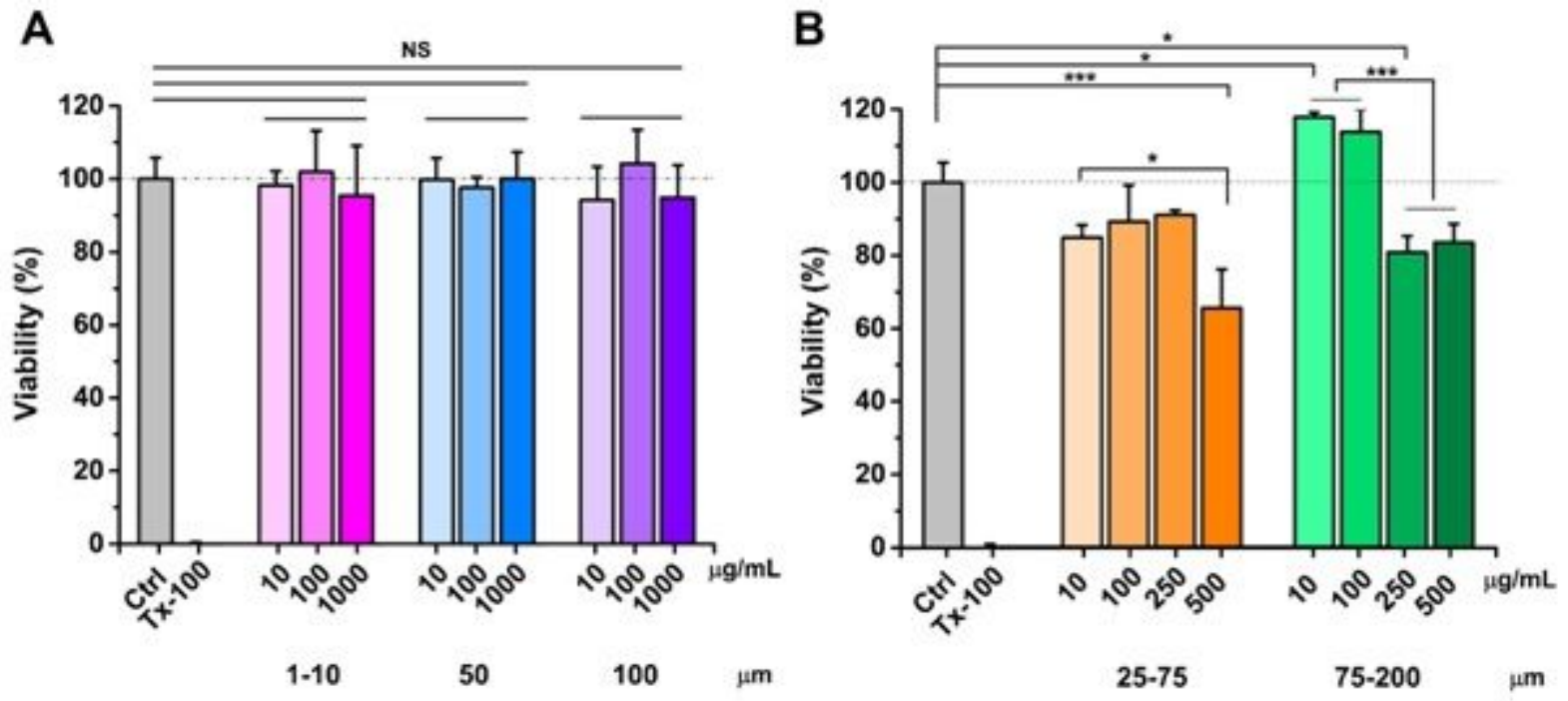

Figure 5

Live/dead ratio of PBMCs upon treatment with (A) HDPE microparticles and (B) LDPE ground particles for $24 \mathrm{~h}$. Statistical analysis was performed by ANOVA followed by Tukey's post hoc test $(n=3)$. NS and * indicate "non-significant" and " $\mathrm{p}<0.05$ " compared to Ctrl (Control), respectively. 

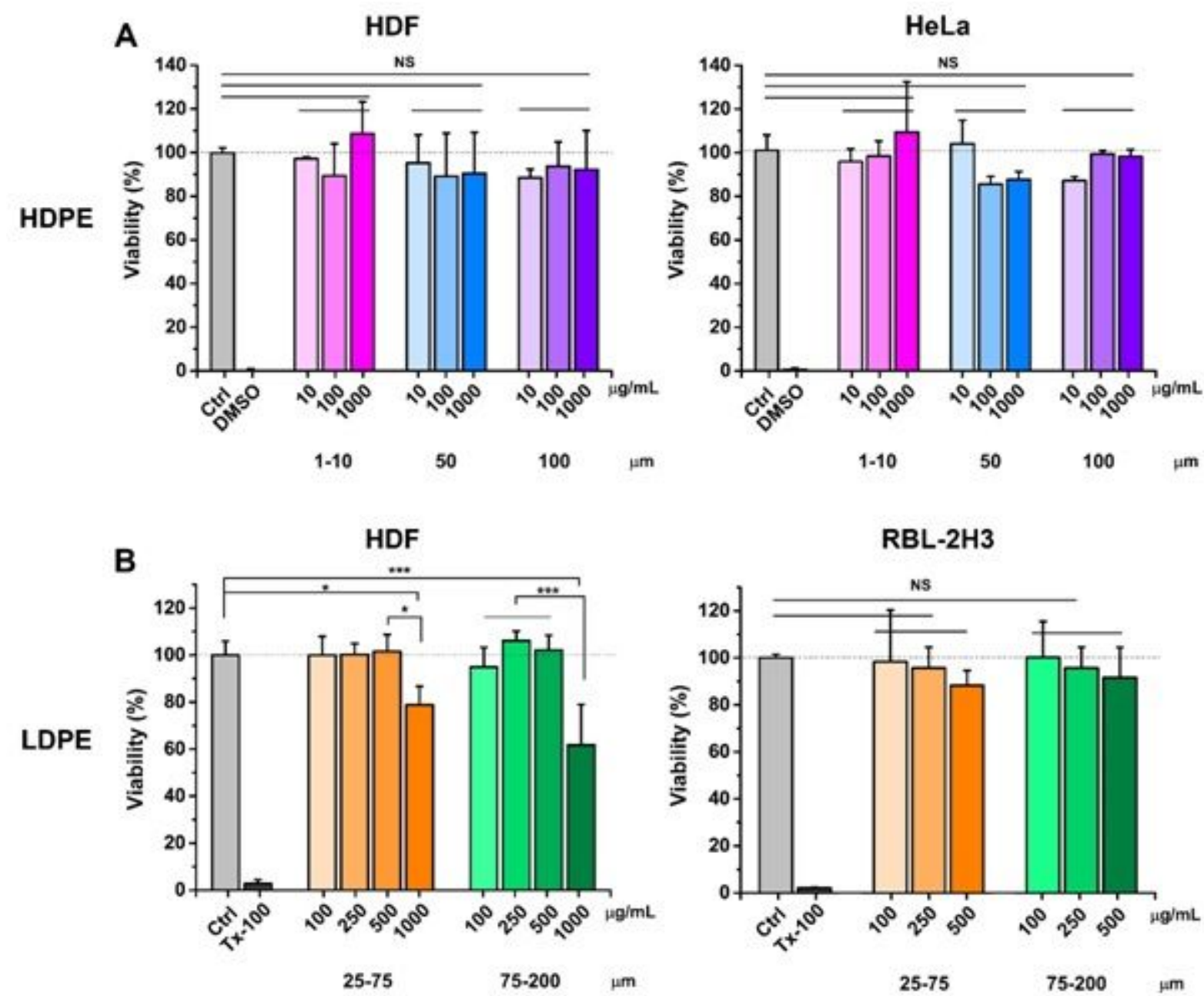

Figure 6

Viability of (A) HDF and HeLa cells in response to HDPE particles, and that of (B) HDF and RBL-2H3 cells in response to LDPE particles. Statistical analysis was performed by ANOVA followed by Tukey's post hoc test $(n=3)$. NS and * indicate "non-significant" and " $p<0.05$ " compared to Ctrl (Control), respectively. 

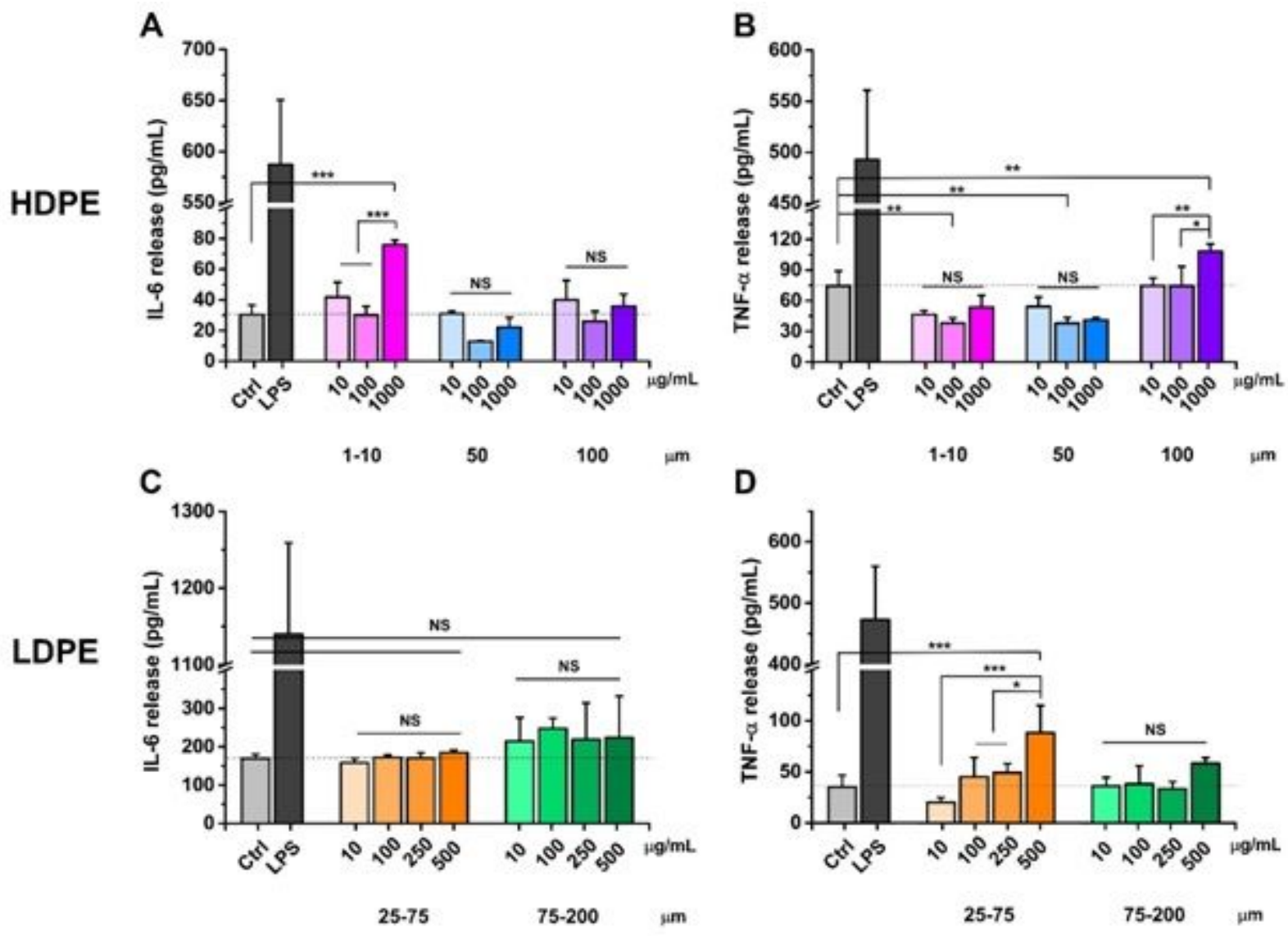

\section{Figure 7}

Cytokine (IL-6 and TNF-a) release from PBMCs after four days of culture with HDPE and LDPE particles at different concentrations. LPS $(5 \mathrm{gg} / \mathrm{mL})$ treated cells were used as a positive control. Statistical analysis was performed by ANOVA followed by Tukey's post hoc test $(n=3)$. NS, *, **, and *** indicate "nonsignificant", " $p<0.05$," " $p<0.01$," and " $p<0.001$," respectively.
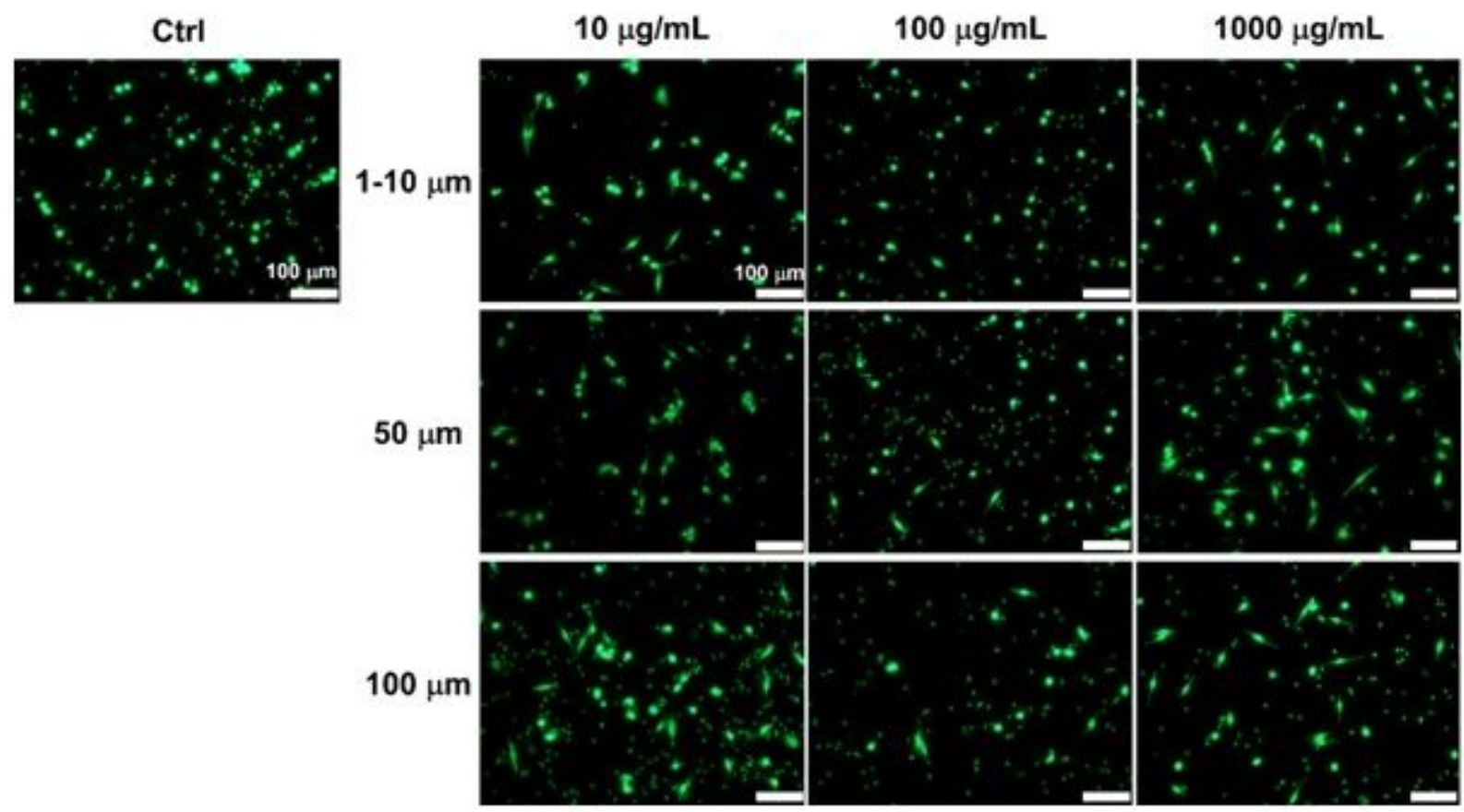


\section{Figure 8}

Fluorescence images of PBMCs after five days of treatment with HDPE particles. Live cells were stained by Calcein AM. The scale bar represents $100 \mathrm{Dm}$.
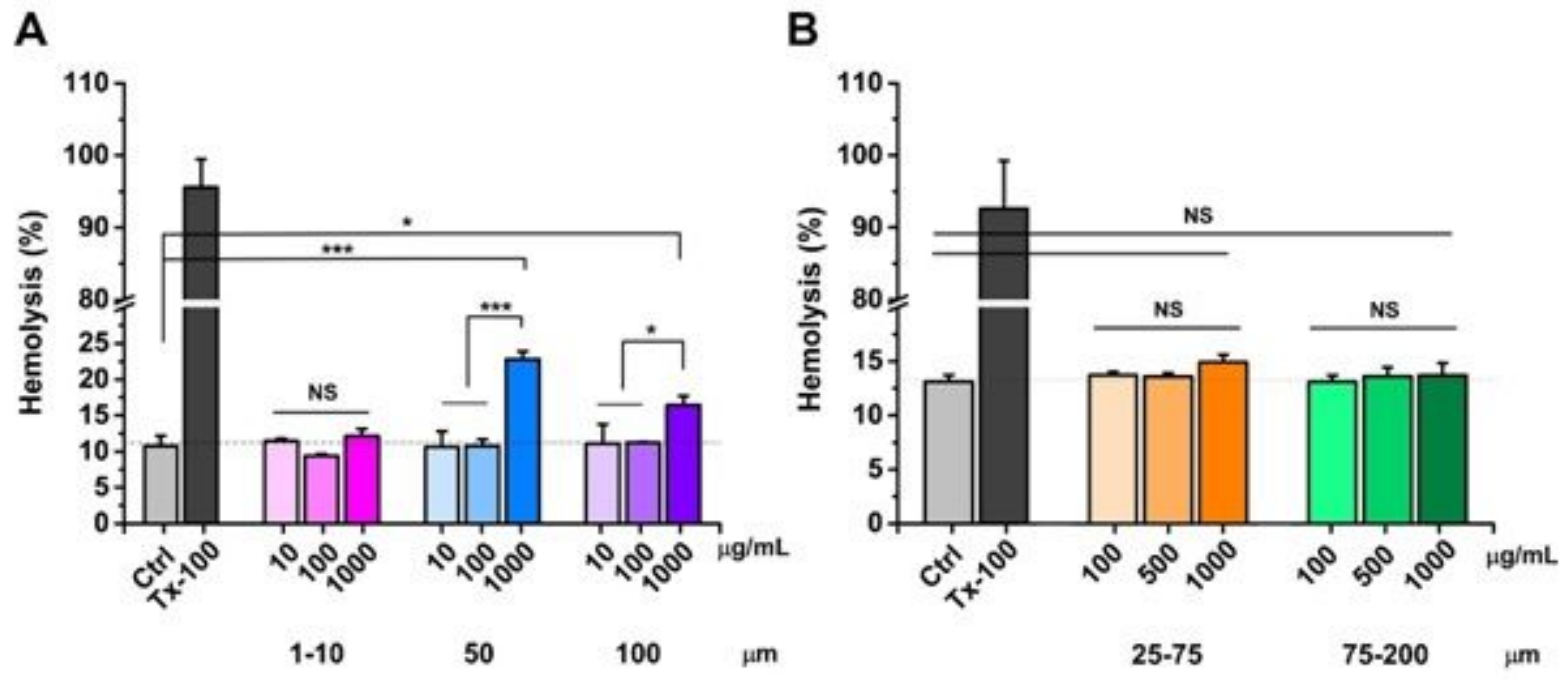

Figure 9

Cytotoxicity of PE particles toward RBCs. Hemolysis ratio in RBCs upon exposure to (A) HDPE and (B) LDPE particles for $1 \mathrm{~h}$. Statistical analysis was performed by ANOVA followed by Tukey's post-hoc test

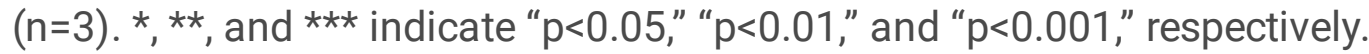

\section{Supplementary Files}

This is a list of supplementary files associated with this preprint. Click to download.

- revisedsupplementary.docx

- Responsetoreviewerparticleandfibretoxicology.docx

- revisedsupplementary.docx

- Responsetoreviewerparticleandfibretoxicology.docx 